\title{
Philosophiques
}

\section{Le livre sur Adler de Soren Kierkegaard}

\section{Hervé-Marie Gicquel}

Volume 12, numéro 2, automne 1985

URI : https://id.erudit.org/iderudit/203290ar

DOI : https://doi.org/10.7202/203290ar

Aller au sommaire du numéro

Éditeur(s)

Société de philosophie du Québec

ISSN

0316-2923 (imprimé)

1492-1391 (numérique)

Découvrir la revue

Citer cet article

Gicquel, H.-M. (1985). Le livre sur Adler de Soren Kierkegaard. Philosophiques, 12(2), 315-362. https://doi.org/10.7202/203290ar

\section{Résumé de l'article}

Le livre sur Adler est un des ouvrages-clés de la pensée kierkegaardienne. En l'écrivant, le penseur danois a pu vérifier pour lui-même la teneur religieuse de sa vocation et la justesse de sa critique contre l'Église et la société modernes. Il présente une investigation approfondie des concepts chrétiens fondamentaux de " révélation », d'« autorité divine » et d'« apôtre » qui démontre que Kierkegaard, loin d'être « subjectiviste », s'est doté d'un savoir objectif, grâce auquel, par ailleurs, il fait voir la nécessité d'une distinction qualitative marquée entre ce qui, d'une part, appartient à l'ordre immanent, c'est-à-dire aux hommes, et ce qui, d'autre part, appartient à l'ordre transcendant, c'est-à-dire à Dieu. Mais Le livre sur Adler est également le résultat d'une étude dialectique et psychologique profonde faite sur un dénommé Adler qui s'est importunément prévalu d'une révélation. Le livre donne donc, dans le détail, les preuves les plus évidentes de sa confusion et en expose les causes réelles: un choc émotif, un manque sérieux de formation conceptuelle chrétienne et d'éducation éthico-religieuse, une mauvaise influence de la philosophie hégélienne. Et tandis qu'il fait voir en quoi Adler est l'épigramme de son époque, il nous fait comprendre le danger, toujours actuel, de rater l'essentiel du phénomène religieux.
Ce document est protégé par la loi sur le droit d'auteur. L'utilisation des services d’Érudit (y compris la reproduction) est assujettie à sa politique d'utilisation que vous pouvez consulter en ligne.

https://apropos.erudit.org/fr/usagers/politique-dutilisation/ 


\title{
LE LIVRE SUR ADLER DE SOREN KIERKEGAARD
}

\author{
par Hervé-Marie Gicquel
}

RÉSUMÉ. Le livre sur Adler est un des ouvrages-clés de la pensée kierkegaardienne. En l'écrivant, le penseur danois a pu vérifier pour lui-même la teneur religieuse de sa vocation et la justesse de sa critique contre l'Église et la société modernes. Il présente une investigation approfondie des concepts chrétiens fondamentaux de " révélation ", d' " autorité divine " et d' " apôtre " qui démontre que Kierkegaard, loin d'être "subjectiviste ", s'est doté d'un savoir objectif, grâce auquel, par ailleurs, il fait voir la nécessité d'une distinction qualitative marquée entre ce qui, d'une part, appartient à l'ordre immanent, c'est-à-dire aux hommes, et ce qui, d'autre part, appartient à l'ordre transcendant, c'est-à-dire à Dieu. Mais Le livre sur Adler est également le résultat d'une étude dialectique et psychologique profonde faite sur un dénommé Adler qui s'est importunément prévalu d'une révélation. Le livre donne donc, dans le détail, les preuves les plus évidentes de sa confusion et en expose les causes réelles : un choc émotif, un manque sérieux de formation conceptuelle chrétienne et d'éducation éthico-religieuse, une mauvaise influence de la philosophie hégélienne. Et tandis qu'il fait voir en quoi Adler est l'épigramme de son époque, il nous fait comprendre le danger, toujours actuel, de rater l'essentiel du phénomène religieux.

ABSTRACT. The book on Adler is one of the key works in Kierkegaard's thought. By writing it the Danish philosopher was able to verify for himself the religious content of his vocation and the accuracy of his critique against the modern church and society. It presents a profound investigation into the fundamental Christian concepts of "revelation", of "divine authority", and of "apostle". The inquiry shows that Kierkegaard is more of an objectivist than a subjectivist because he pronounced qualitative distinction between what belongs to the immanent order, i.e. to men, and what belongs to the transcendal order, i.e. to God. But The book on Adler is also the result of a profound dialectical and psychological study performed on a certain Adler who pactatively gratified himself whith a revelation. The book, 
then, gives in details the most obvious proofs of his confusion and gives an account of its real causes : an emotionnal shock, a lack in both a Christian conceptual formation and an ethicoreligious education, and the negative influence of Hegelian philosophy. While it sees in Adler the epitome of his era, it makes us understand the continual danger of missing what is essential in religious phenomena.

« Aucun texte de Kierkegaard n'a été autant retravaillé que Le livre sur Adler, et cependant ce texte demeura inédit du vivant de son auteur ${ }^{1}$. L'ouvrage, en effet, fut constamment remanié par Kierkegaard de 1846 à 1855 , et une fois seulement un court extrait, intitulé Sur la différence entre un génie et un apôtre, parut dans les Deux petits traités étbico-religieux qu'il publia en $1849^{2}$. C'est déjà dire toute l'importance que le penseur danois accordait à ce livre, qui contient par ailleurs l'essence même de sa pensée, mais qui, malheureusement, en raison de sa très récente traduction en 1983, par Else-Marie Jacquet-Tisseau, reste encore fort peu connu en milieu francophone.

Or la connaissance du Livre sur Adler est à maints égards essentielle pour quiconque entend donner une juste interprétation de l'œuvre kierkegaardienne ; premièrement parce que sa rédaction a permis à Kierkegaard de se confirmer dans sa vocation d'écrivain religieux et d'éveilleur de conscience ; deuxièmement, parce qu'elle lui a donné l'occasion d'approfondir certains concepts chrétiens fondamentaux et de se doter ainsi d'un vocabulaire objectif nécessaire à toute philosophie de la religion ; troisièmement, parce que le livre produit un exemple remarquable de ce que peut être sa dialectique appliquée à un cas particulier.

1. Oeuvres complètes de Soren Kierkegaard, traduction du danois par Else-Marie Jacquet-Tisseau, Paris, Éditions de l'Orante, tome XII, voir l'avertissement de l'éditeur, p. xxiv. Le livre sur Adler (en danois : Bogen om Adler) est le titre sous lequel les différents remaniements ont été réunis dans les Papirer. Le dernier article paru en français sur Le livre sur Adler, intitulé " Le Général, l'Individu, l'Exception, Kierkegaard et le pasteur Adler ", est celui d'André Clair dans la Revue de Sciences philosophique et théologique, 68 (1984), p. 212-228. En voici le résumé : "En réfléchissant dans son Livre sur Adler sur le statut de l'homme exceptionnel, Kierkegaard explique que cet homme doit d'abord se référer à la généralité et refuser l'exaltation délirante. C'est alors à partir d'un rapport opposé à la généralité que se distinguent l'homme ordinaire (vivant dans l'immanence) et l'homme extraordinaire, élu de Dieu, prêt à vivre jusqu'au martyre la relation et la tension entre la généralité et l'appel singulier ». Les sigles $0 . C$., utilisés à la suite, désigneront une référence aux Oeuvres complètes, édition française.

2. O.C., XVI. 
En tant qu'auteur essentiellement religieux, dont la mission était de " rendre attentif au donné religieux " et de permettre à l'Individu de « devenir chrétien ${ }^{3}$, Kierkegaard ne pouvait, certes, qu'être directement concerné par le cas du Magister A.P. Adler, lequel s'était attiré l'intérêt du public et la réprobation des autorités religieuses. L'affaire de ce pasteur danois, qui affirmait avoir reçu une « révélation » au cours d'une certaine nuit de l'hiver 1842, avait en effet soulevé tout un émoi parmi la petite population de Copenhague, avant d'être portée devant les autorités ecclésiastiques en avril 1845 pour y être débattue. Vivement intéressé par cette polémique, Kierkegaard s'était empressé d'acheter tous les volumes qu'Adler avait publiés à la suite de son extraordinaire expérience, mais leur lecture raviva aussitôt chez lui un grand nombre d'inquiétudes et de problèmes religieux qui, toute sa vie, n'avaient jamais cessé de le hanter : lui qui se faisait le défenseur hardi du christianisme était-il réellement un missionnaire ou avait-il étourdiment, comme Adler, succombé à une tentation? Était-il sûr de sa vocation ? N'avait-il pas plutôt confondu ce qu'il prenait pour un appel avec une simple exaltation subjective ? Plus que jamais l'affaire Adler avait ranimé en lui la question existentielle, qui primait à ses yeux sur toutes les autres, et secoué son âme en soulevant en elle d'intenses et profondes préoccupations vocationnelles. Doué d'une probité intellectuelle peu commune, Kierkegaard, au fur et à mesure qu'il démystifiait la révélation du dit Magister, se demandait de plus en plus s'il était vraiment cet être d'exception qu'il croyait être, cette sorte de nouveau Socrate fervent du christianisme qui, au risque d'être bafoué, aurait jeté toute sa différence individuelle et religieuse au sein de sa génération. Se situant, par conséquent, au cœur même de ses préoccupations existentielles, l'histoire d'Adler fut donc, pour le penseur danois, l'occasion inespérée de vérifier pour son propre compte la teneur religieuse de sa vocation. Tel a dû être en tout cas son premier intérêt, à l'heure où il sentait se dessiner sa polémique prochaine avec l'Église établie ${ }^{4}$.

3. Sur mon auvre d'écrivain, O.C., XVII, p. 270. Le mot "Individu " traduit ici l'expression danoise " den Enkelte " employée dans Papirer VIII ${ }^{2}$ B 27.

4. Kierkegaard entra ouvertement en guerre contre l'Église établie à partir de l'hiver 1854 . Mynster, alors évêque de Copenhague, avec lequel Kierkegaard n'avait guère d'atomes crochus, était décédé en janvier la même année. Son éloge funèbre avait été prononcé par 
Outre l'influence historique déterminante qu'eut cette affaire sur l'existence de Kierkegaard, il faut aussi considérer l'orientation assez singulière qu'elle donna au contenu de son ouvrage. C'est que Le livre sur Adler est en réalité un important écrit d'édification, "une œuvre, écrit Kierkegaard, véritablement édifiante", mais «- pour quiconque a les qualités requises pour se laisser édifier par une lecture par ailleurs astreignante ${ }^{5}$. Ce livre, nous avertit-il, " ne pourra, essentiellement, être lu que par des théologiens et, parmi ces derniers, n'intéressera essentiellement que l'Individu ${ }^{6}$. Kierkegaard savait, à l'évidence, qu'il écrirait pour une petite sélection de lecteurs, et parmi ceux-ci pour l'Individu qui saurait satisfaire à ses exigences. $\grave{A}$ ce dernier cependant il promettait une édification particulière : car, nous assure-t-il, «ce livre, je m'en porte garant, l'éclairera et lui donnera une souplesse dans la pratique de certains concepts dogmatiques ${ }^{7}$. A cet égard, en effet, Le livre sur Adler se présente fondamentalement comme une investigation approfondie des concepts chrétiens de "révélation " ou d'« autorité », divine et apostolique, concepts dogmatiques sus-

celui qui serait alors son successeur à l'évêché, le professeur de théologie Hans Martensen. Dans son oraison funèbre, Martensen avait parlé de Mynster comme d'un * témoin de la vérité ", affirmation à laquelle Kierkegaard avait réagi avec force en faisant aussitôt paraître un article intitulé "L'évêque Mynster a-t-il été un témoin de la vérité ?" (voir $0 . C$., XIX, p. 3. L'article est traduit en entier par Else-Marie Jacquet-Tisseau), dans lequel il dénonçait le ministère de Mynster comme celui d'un individu qui identifiait le Christ à l'ordre établi et qui faisait consister sa vocation dans l'exercice d'une charge publique. Pour le penseur danois, en effet, l'évêque Mynster n'avait été en aucun moment comparable à un véritable " témoin de la vérité ", lequel témoin est plutôt initié aux combats intérieurs et, confiant en l'aide divine, ne désavoue jamais sa vocation pour faire des compromis avec le reste des hommes ou par crainte de provoquer un scandale. Cependant on peut aussi retracer les germes de cette polémique dans des écrits antérieurs à 1854 , comme dans Crainte et tremblement $(O . C$., V), paru en 1846, ou comme dans le Post-Scriptum definitif aux Miettes philosopbiques $(O . C$, XI), paru en 1846, deux ceuvres pseudonymes dans lesquelles Kierkegaard, en posant la foi comme un paradoxe invraisemblable pour la pensée, s'élève contre la théologie et l'exégèse modernes qui, toutes pénétrées de philosophie rationalisante, cherchent le plus possible à rendre le christianisme cohérent, intelligible et vraisemblable. Ou encore comme dans ses Deux petits traités étbico-religieux (O.C., XVI) de communication directe, parus en 1849, où l'on peut trouver des écrits aux titres assez annonciateurs, dont "Le droit de mourir pour la vérité " et * Sur la différence entre un génie et un apôtre »; comme, enfin, dans cet autre important ouvrage d'édification signé du pseudonyme AntiClimacus, paru en 1850 et intitulé L'École du Christianisme, qui, d'une façon bien irrévérencieuse, compare la chrétienté établie à une institution stagnante et sans devenir chrétien (O.C., XVII).

5. Le livre sur Adler, O.C., XII. Annexes. I. Projets d'avant-propos de $1847 ; 2$. Deuxième projet, p. 236.

6. Ibid., 1. Premier projet d'avant-ptopos, p. 231.

7. Ibid., 2. Deuxième projer, p. 233. Voir Papirer VIII ${ }^{2}$ B 27. 
ceptibles d'être opposés, dans toute la force de leur ampleur qualitative, à n'importe quelle tentative injustifiée pour se les accaparer.

Au fond, ce livre analyse le concept de révélation, analyse ce qu'il faut entendre par "être appelé par une révélation " du point de vue de l'éthique ; il examine comment celui qui l'a reçue se rapporte à la génération, au général, et comment nous, les autres, nous rapportons à lui ; il examine toutes les confusions dont le concept de révélation est victime à notre époque confuse. Ou, ce qui revient au même, tout le livre est, au fond, une analyse du concept d'autorité : que signifie « avoir une autorité divine $" ?^{8}$

Partant, Le livre sur Adler dénombre tous les risques que court le subjectivisme religieux lorsque, comme chez Adler, il n'a pas d'autre fondement qu'une expérience individuelle, si extraordinaire qu'elle puisse paraître. Cette observation, banale en apparence, est pourtant d'une certaine conséquence, puisqu'elle s'inscrit en faux contre une tradition encore profondément enracinée dans nos milieux francophones qui se plaît à présenter Kierkegaard comme un "subjectiviste " qui aurait transformé la religion en philosophie. Une telle accusation pourtant ne peut avoir de sens en ce qui concerne un penseur qui s'est si radicalement prononcé contre le subjectivisme en fondant sa critique sur la base de concepts dogmatiques rigoureux'. De plus, ne se

8. Ibid., 2. Deuxième projet, p. 234. Voir Papirer VIII ${ }^{2}$ B 27.

9. La plupart de ces accusations portées contre Kierkegaard partent de l'analyse d'œuvres de communication indirecte, telles que le Post-Scriptum de 1846, dans lesquelles le penseur danois fait parler des personnages qui ne sont pas nécessairement représentatifs de sa propre pensée religieuse. Par ailleurs le cas du fameux Post-Scriptum, où Climacus soutient, d'une façon qui frôle presque l'irrationalisme, que " la subjectivité est la vérité ", et auquel on se fie ordinairement pour affirmer que Kierkegaard a renié le savoir religieux, s'explique à la lumière d'un éclairage contextuel. Plus porté à captiver la pensée qu'à la convaincre, le penseur religieux, en réaction contre l'excès d'objectivité des temps modernes et toujours soucieux d'un certain équilibre dans l'état des esprits, a par stratégie, préféré insister davantage sur certains aspects de l'existence humaine autres que la pensée (subjectivité, intériorité, moralité, etc.) dans le but de faire mieux comprendre que ces aspects n'étaient pas uniquement des objets de connaissance mais aussi des expériences vécues. C'est aussi à cause de cette préoccupation que Kierkegaard passe pour le père de l'existentialisme contemporain. Au reste, il est également essentiel de noter que, dès 1834 , donc bien avant la rédaction du Post-Scriptum, Kierkegaard avait déjà pris conscience de la nécessité d'un savoir dogmatique, par conséquent objectif, tiré de l'activité du Christ (voir Papirer I A 27 dans Soren Kierkegaard's Journals and Papers, vol. II, no. 412, ed. \& transl. by Howard V. and Edna H. Hong, Indiana University Press, Bloomington and London, C1967). Cette même prise de conscience va en s'accentuant dans les écrits de communication directe subséquents, en sorte que l'idée d'une évolution de la pensée de Kierkegaard en ce qui a trait à la nécessité d'un savoir conceptuel et dogmatique reste encore à envisager. Dans les 
limitant pas à dénoncer seulement le subjectivisme religieux, Le livre sur Adler, tandis qu'il fait transparaître à travers sa critique la confusion de son époque, révèle aussi le danger qui guette chaque Individu de verser dans un christianisme caduque et édulcoré. Il propose par conséquent le seul remẹde apte à protéger la sphère religieuse des atteintes du mal moderne en indiquant par quelles voies intérieures l'on peut devenir authentiquement chrétien. En ce sens, certes, un véritable apôtre est un modèle de premier ordre, et l'on devine, du reste, et sous bien des aspects, quelle leçon il est possible de tirer aujourd'hui d'un contenu aussi universel, à une époque où les manifestations extérieures de la religion sont devenues si imposantes et où l'exaltation subjective, voire même sentimentale, gagne parfois les croyants $^{10}$.

Toutefois, il faut souligner ici que Kierkegaard se défendait bien d'être un apôtre, consçient qu'il était de l'importance d'une telle catégorie dogmatique et des responsabilités éthico-religieuses que celle-ci impliquait. Dans ses ouvrages de communication directe, c'est même avec beaucoup d'insistance qu'il se dit et

Trois discours édifiants de 1844 (O.C., VI, p. 215-231), notamment, on retrouve un texte d'une splendide facture, intitulé "Souviens-toi de ton Créateur pendant les jours de ta jeunesse ", qui débute en distinguant les " vérités indifférentes " des " vérités soucieuses ". Cette distinction très nette entre, d'ụne part, des vérițés objecțives, également recevables, applicables de manière générale à toutes les circonstances et témoignant d'une véritable compréhension, et, d'autre part, des vérités éprouvées, non indifférentes à la subjectivité et faisant plutôt partie intégrante du langage du cœur que de celui impératif de la pensée, marque bien les débuts, chez le penseur danois, d'une conception objective de la vérité. Plus tard, en 1846, Le livre sur Ádler établit une ligne de démarçation très précise entre les concepts d' " autorité divine " et de " confusion religieuse ", d' " ạôtrẹ » et de " génie "., tandis qu'en 1847, dans sa Dialectique de la communication éthique et éthico-religieuse (O.C., XIV), Kierkegaard s'explique enfin sụr cette nécessité d'une connaissance conceptuẹlle chrétienne en tant qu'annonce directe des grands actes de Dieu rapportés par la Révélation. - Comment, en ce cas, peut-on encore parler de subjectivisme ? Ce manquẹ de compréhension du pensẹur danois ne peut provenir que d'une méconnaissançe des œuvres de communication à type direct, erreur souvent causée par le passé par des retards dans la traduction. Quoi qu'il en soit, il sera toujours utile de consultẹ à çe sujet l'excelleṇt article de Jean Caron, "Dialectique de la communication chez Kierkegaard », dans Philosophiques, oct. 1976, vol. III, no 2, p. 167 et celui de Maurice Carignan, en introduction, « L'ambivalẹnce de la réflexion seḷon Kierkegaard ", dans Église et Théologie, 12 (1981), p. 223225. Quant à savoir si Kierkegaard a transformé la religion en philosophie, la question à notre avis ne se pose pas pour un auteur qui a passé toute sa vie à̀ rețranchẹr de la sphère immanente ce quị a toujours apparțenu à la sphère transcendante.

10. En ce qui concerne la pertinence de l'œuvre kierkegaardienne pour nos temps contemporains, voir l'article de Michel Cornu, "Actualité de Kierkegaard ", dans Revue de Théologie et. de Philosophie, 3-21, 1971, p. 428-437. 


\section{répète qu'il est « sans autorité », tant dàns chaque avant-propos de ses Discours édifiants que dans le livre sur Adler, ou que dans son Point de vue explicatif sur mon cuvre d'écrivain ${ }^{11}$. Telle une}

11. Dans l'avant-propos de ses Deux discours édifiants de 1843, il écrit : "Quoique ce petit livre (appelé « Discours " et non "Sermons ", car l'auteur n'a pas d'autorité pour prêcher ; "Discours édifiants " et non «Discours pour l'édification", car l'orateur ne prétend nullement être un maître) n'ait certes d'autre désir que d'être ce qu'il est, c'est-à-dire superflu, et ne souhaite rien tant que de demeurer dans le secret comme il y eśt né . . . " (0.C., VI, p. 5). La même version de cette rétractation est reprise quasi littéralement dans chaque avant-propos de tous ses Discours édifiants jusqu'en 1844 (comparer p. 53, p. 101, p. 165 , p. 213 et p. 269). Tandis qu'il écrit dans Le livre sur Adler (1846-1855) : "Peut-être tel ou tel lecteur se rappellera-t-il que, parlant de moi en tant qu'auteur, j'ai toujours employé l'expression "sans autorité " et j'ai tant insisté sur cette expression qu' " on en trouve la formule répétée dans chaque avant-propos ". Si donc je n'ai pas fait œuvre utile en tant qu'écrivain, j'ai du moins fait tout mon possible pour éviter d'altérer ce qu'il y a de plus élevé et de plus sacré. Je suis un pauvre homme, un particulier ; si comme d'aucuns le croient, j'ai quelque génie, je dirai : qu'importe ! Mais sur le plan qualitatif un apôtre est à tout jamais aussi différent de moi que du plus grand génie de tous les temps, comme de l'homme le plus sot qui a jamais vécu » (O.C., XII, p. 141, remarque no *). Mais c'est encore dans son Point de vue explicatif sur mon auvre d'écrivain, écrit en 1848, quoique jamais publié de son vivant, que Kierkegaard se découvre le mieux : "Suis-je peut-être " apôtre "? Abomination ! Je n'ai jamais donné sujet à une pareille interprétation ; je ne suis qu'un pauvre homme insignifiant. Suis-je donc un maître, un éducateur? Par davantage ; je suis celui qui a lui-même été éduqué et dont l'œuvre exprime la discipline conduisant au devenir chrétien : tandis et puisque cette éducation pèse sur moi, je fais à mon tour pression sur l'époque, mais loin d'être un maître, je ne suis qu'un condisciple " (O.C., XVI, p. 53). Cependant certains commentateurs prétendent, comme par exemple T. H. Croxall (voir "Kierkegaard, On Authority " in The Hibbert Journal, XLVIII, oct. 1979, p. 146) que Kierkegaard, tout comme Adler, se croyait "Extraordinaire" ; tandis que d'autres, comme André Clair et Nelly Viallaneix, qui sont bien au courant de son humilité, n'hésitent pas pour autant à le caractériser d' "Individu exceptionnel " ou de "prophète de la modernité " (voir l'article de Clair, déjà mentionné, p. 228, et celui de madame Viallaneix, "Kierkegaard, prophète de la modernité " paru dans le Bulletin de la Société de l'bistoire du Protestantisme français, tome CXXVII, juillet-août-septembre 1981, Paris, 1981, p. 363-383). Si sans doute il est vrai, ainsi que le fait judicieusement remarquer André Clair, que Kierkegaard, " en produisant une cuvre aussi exceptionnelle (. . . ) a fort bien saisi la distance entre l'appel et la réalisation, la différence entre le fait de se rapporter au paradoxe et l'acte de l'accomplir ; et, en accentuant cette distinction, (. . .) a compris que la tâche est de vivre dans une infinie tension ", il n'en découle pas nécessairement qu'on puisse le prendre, implicitement ou non, pour un apôtre. Le problème qui est certes le plus embarrassant est que la définition que Kierkegaard donne de l'apôtre présente une similitude frappante avec l'exemple de sa vie, de son humilité à se dire sans autorité et sans foi, en passant par son soulèvement contre l'Église établie, jusqu'à son sacrifice et sa souffrance, consentis pour lui-même et pour la généralité. Pourtant il reste un point qui, à ce stade des études kierkegaardiennes, ne semble pas avoir été éclairci ; à savoir justement s'il n'y a pas une distinction à concevoir entre l'Individu dit Extraordinaire ou dit Exceptionnel et l'Individuapôtre, ce peut être en dépit de tous les parallèles déroutants que Kierkegaard a faits ou que nous puissions faire entre ces deux notions. Notre avis est que si l'apôtre est un Extraordinaire, l'inverse ne s'ensuit pas avec évidence, parce que l'apôtre jamais ne s'abaissera, comme Kierkegaard l'a fait avec une génialité remarquable à travers toute son œuvre, à vouloir donner son point de vue explicatif sur sa différence qualitative, éternelle et paradoxale. L'apôtre, en effet, n'ira pas jusqu'à défendre aussi clairement sa différence ; il continuera d'agir. En ce sens, et aussi pour apporter une source de référence nouvelle 
créature qui, devant Dieu, réalise très humblement sa contingence, il connaissait sa dépendance au christianisme ; mais si, un peu comme Pascal, qui passe pour avoir quelquefois malmené l'homme par amour, il a été réactionnaire, ce ne fut jamais qu'en mettant sans ménagement son génie au service du Christ et en rendant à Dieu ce qui appartient à Dieu. Aussi Le livre sur Adler n'est-il point l'œuvre d'un apôtre mais l'œuvre d'un condisciple, d'un Socrate moderne et religieux qui a voulu défendre certains concepts chrétiens fondamentaux contre l'intrusion des vanités et des libres spéculations humaines.

Cependant Le livre sur Adler, s'il a eu un rôle à jouer dans l'existence de Kierkegaard en le confirmant dans sa vocation et en lui permettant de se rendre compte davantage de la nécessité d'un savoir conceptuel chrétien, raconte avant tout la confusion d'un pasteur de campagne qui croit avoir reçu une révélation. Il en fait donc un exposé très clair et très précis. Né en 1812 et mort en 1869, Adolph Peter Adler, pasteur danois d'une grande culture, connaît une histoire relativement courte mais fort chargée en événements. En 1846, à l'Université de Copenhague, Adler passe son examen supérieur de théologie. De 1837 à 1841, s'étant familiarisé avec la philosophie allemande, et surtout hégélienne, il remet une thèse sur la " subjectivité ", reçoit le titre de Magister Artium et tient, toujours à l'Université de Copenhague, une série de conférences publiques sur la logique spéculative de Hegel dont les retentissants succès l'encouragent à entreprendre la rédaction d'un ouvrage de vulgarisation sur le sujet. Bref, en quatre ans à peine, Adler, disciple inconditionnel de Hegel, est devenu un conférencier très couru à Copenhague. Or, c'est au moment où il commence à goûter les fruits de sa réussite, c'est-à-dire en 1841 , que les autorités ecclésiastiques décident de le nommer pasteur de Hasle et Rutsker, deux bien paisibles paroisses de l'île de Bornholm, à des lieues de son habituel univers intellectuel. Brusquement, tout change dans sa vie. Une nuit de décembre 1842, phénomène incroyable,

sur cette question, nous verrions Kierkegaard comme un génie religieux extraordinaire dont le charisme dériverait d'une communication divine de l'essence de Dieu (c.-à-d. le Christ), un peu à la manière dont Max Scheler le classifie dans un ouvrage aujourd'hui oublié: Le Saint, le Génie, le Héros (traduit de l'allemand par Émile Marmy, Paris-Lyon, Emmanuel Vitte, Éditeur, 1958. Collection animus et anima, p. 43-44), c'est-à-dire parmi les types-limites de religieux purs comme Socrate et Pascal, à la frontière entre les apôtres et les sages. 
Adler reçoit une « révélation ». Dans l'avant-propos d'un ouvrage qu'il publie dès 1843 , sous le titre Quelques sermons, il raconte de la manière suivante son extraordinaire expérience : au milieu de la nuit (alors qu'il avait travaillé toute la veille à son étude sur la logique subjective), il fut soudainement réveillé par un bruit abominable; le Sauveur en personne lui ordonna de se lever pour prendre en notes une doctrine nouvelle, exigeant de lui qu'il brûle ses propres écrits et qu'il s'en tienne désormais à la Bible. Puis, parlant de son ouvrage, Adler conclut son avantpropos en affirmant : «Les Sermons et les Discours, depuis le no VI jusqu'au dernier, sont, je le sais, écrits avec la grâce concomitante de Jésus ; aussi n'ai-je été qu'un instrument ${ }^{12}$. Mais parce que le Magister Adler prétendait être devenu le dépositaire d'une « doctrine nouvelle », dont le Sauveur en personne (entendons directement) lui aurait fait part, les autorités ecclésiastiques crurent plus prudent, dès le 19 janvier 1844, de le suspendre de sa charge pastorale. Du coup le Magister s'attire l'estime de la population et des pétitions sont même signées en faveur de son maintien. Bizarrement, c'est Adler lui-même qui réclame la tenue d'une commission d'enquête dont l'Église danoise retardera volontairement la constitution jusqu'au 29 avril 1845 . Enfin, le 10 mai 1845, Adler paraît devant la commission qui lui pose des questions très précises sur sa "révélation ». Mais, un peu plus tard, le 5 juillet, Adler, qui cherche à revenir sur ses explicaitons précédentes, déjà fort compromettantes, fait de nouvelles déclarations dans lesquelles il oublie et altère profondément les termes de sa position initiale ; ceci sans pour autant vouloir faire mine de se rétracter. Nonobstant, sa déposition est notée, telle

12. Le texte de l'avant-propos est traduit dans Le livre sur Adler, O.C., XII, sous la rubrique : Textes de A.P. Adler tirés de Quelques sermons (1843), Annexes V, p. 251. En voici la traduction : "Au mois de décembre, l'année dernière, j'avais presque terminé une étude que je me proposais d'intituler Cours populaires sur la logique subjective. Ma propre pensée avait plongé en elle-même et, avec une connaissance superficielle de la Bible, entrepris d'expliquer la création et le christianisme. Un soir que je développais justement l'origine du mal, j'entrevis comme dans un éclair que tout relève, non de la pensée, mais de l'Esprit et qu'il y avait un esprit malin. Cette même nuit un bruit abominable fondit sur notre chambre. Alors le Sauveur m'ordonna de me lever et d'aller noter les mots suivants : " Les premiers hommes auraient pu avoir une vie éternelle; en effet, quand la pensée unit l'Esprit de Dieu au corps, la vie est éternelle . . . " etc. Puis Jésus m'ordonna de brûler mes propres écrits et de m'en tenir désormais à la bible. Les Sermons et les Discours, depuis le no VI jusqu'au dernier, sont, je le sais, écrits avec la grâce concomitante de Jésus ; aussi n'ai-je été qu'un instrument ". Hasle, le 18 juin 1843. 
quelle, le 13 septembre de la même année. Aussitôt, et sans même s'interroger sur les aveux contenus dans sa déposition, Adler, toujours dans un état d'exaltation, édite toutes les pièces relatives à l'enquête, avec tous les détails de son interrogatoire. Puis, de 1846 à 1855, dans la même ligne d'esprit, il publie encore plusieurs essais théologiques et des poèmes.

Sans doute toute cette histoire ne vaudrait-elle pas la peine d'être racontée si Le livre sur Adler ne constituait pas justement l'un des meilleurs exemples kierkegaardiens de la dialectique appliquée à un cas particulier. Rappelons simplement que, pour Kierkegaard, la dialectique agit avant tout dans le sens d'une expérience vécue consistant dans l'engagement et dans la décision, exigeant de la liberté une réduplication (assimilation) et exprimant concrètement le rapport de l'Individu à la vérité comme passion inconditionnelle de la subjectivité pour l'infini. Aussi notre brève introduction historique trouvera-t-elle son utilité, comme on le verra par la suite, puisque c'est au moyen de cette même dialectique existentielle que le penseur danois a entrepris son analyse du " cas Adler ".

Mais quelle fut donc la méthode d'application de cette dialectique à l'analyse des écrits d'Adler ? Il faut dire d'abord que, ce qui, en tant que critique, a particulièrement intéressé Kierkegaard dans l'œuvre d'Adler, ce ne sont pas tant ses qualités esthétiques, par ailleurs peu exceptionnelles, que le problème fondamental qu'en vérité elle soulève, à savoir si Dieu a vraiment parlé à l'auteur qui prétend avoir écrit sous sa dictée. Toutefois une question très sérieuse se posait : - et si tout à coup Adler était vraiment un apôtre, s'il avait réellement reçu une révélation, de quel droit alors pourrait-on remettre en question son autorité divine ? En ce cas, dit Kierkegaard, et parce qu'Adler serait alors un envoyé de Dieu dont on ne pourrait contredire la parole, il faut appliquer la méthode dialectique en tenant compte des observations suivantes : ne rien affirmer ou infirmer « directement " à propos des affirmations d'Adler, tant que la preuve de sa confusion ou celle de son autorité n'a pas été établie à partir de ses propres allégations. Aussi tout le but de la méthode dialectique a-t-il été de prouver la fausseté de cette révélation, non sur la base des affirmations d'une tierce personne extérieure aux faits (ce qu'eût été Kierkegaard ou ce 
que nous eussions été), mais bien « indirectement ", c'est-àdire sur la base des propos tenus par le seul intéressé et pouvant contribuer à l'accuser lui-même. Le fait de la confusion d'Adler devenait alors certain une fois qu'il était démontré $e$ concessis que, finalement, il ne se comprenait pas, n'était pas en accord avec lui-même et ne croyait pas personnellement qu'une révélation lui avait été départie ${ }^{13}$.

En tenant compte de ces recommandations méthodologiques concernant le mode d'application de la dialectique, nous nous sommes proposé, à l'instar de Kierkegaard, de présenter d'abord brièvement l'exposé des preuves les plus évidentes de la confusion d'Adler avant d'en donner les causes réelles. Le compte rendu détaillé des résultats de cette analyse dialectique, en tant que preuves et causes de la confusion d'Adler, fait l'originalité de ce présent article.

Tel que nous allons le considérer à la suite de Kierkegaard, l'ensemble des propos tenus par Adler sur sa " révélation " peut se décomposer en quatre types de déclarations. Les trois premiers types proviennent des Documents concernant ma suspension et ma déposition qu'Adler fit paraître en 1845, aussitôt après la notification de sa déposition ${ }^{14}$. Nous présenterons dans l'ordre chro-

13. Le livre sur Adler, O.C. XII, p. 93, note 193. Concernant toutes ces considérations de méthode, l'éditeur fait remarquer que " dans la version 2, reprise par la version 3, Kierkegaard a ajouté ici sous le titre : "Méthode suivie " le texte suivant : "Nous avons devant nous un homme qui prétend avoir eu une révélation au cours de laquelle il a reçu une doctrine du Sauveur. Un autre n'a le droit de nier directement cette affirmation (à moins de n'avoir aucun sens dialectique) que devant la preuve directe de la folie au sens direct de l'intéressé. Tel n'est pas le cas d'Adler. Cette déclaration qu'il a faite concernant lui-même le place jusqu'à nouvel ordre plus haut que tout autre qui, s'il veut se rapporter à Adler, doit ou bien se soumettre en croyant à son autorité divine, ou bien s'abstenir de toute affirmation comme de toute négation. C'est cette dernière position, sceptique, qu'adopte le présent examen ; mais cette discussion e concessis, tout en s'abstenant de rien affirmer ni infirmer directement, prête pourtant une écoute attentive aux propres allégations du Mag. Adler pour y découvrir s'il ne se comprend pas, s'il n'est pas en accord avec lui-même (sibi constans) et avec la déclaration qu'il a faite à son propre sujet. À cette fin, nous examinerons : 1) Les réponses d'Adler, 2) ses quatre derniers livres ". "

14. Kierkegaard s'est rendu acquéreur de tous les ouvrages écrits par Adler sur sa prétendue révélation. C'est scrupuleusement qu'il a donc examiné les éditions des Quelques sermons (1845) et des Documents concernant ma suspension et ma déposition (1845); puis celles des Quelques poèmes, des Études et exemples, de Essai de bref exposé systématique du christianisme constdéré dans sa logique et de Études théologiques, toutes de 1846. Pour la liste complète des ceuvres d'Adler, voir Le livre sur Adler, O.C., XII, Bibliographie d'A.P. Adler, Annexes VII, p. 254. Pour les titres des livres de la bibliothèque de Kierkegaard en vente après sa mort, consulter l'édition de H.P. Rohde, The auctionneer's sales record of the library of Sфren Kierkegaard (Auktionsprotokal over S. Kierkegaards Bogsamling), The Royal Library (Det 
nologique ces trois premiers types de déclarations avec les principales remarques que Kierkegaard fait à leur propos.

a) Le premier type est celui des déclarations qu'Adler a faites dans l'avant-propos des Sermons de 1843 et constituant sa position fondamentale. C'est " pour apporter le maximum de précision à toute cette affaire et permettre au lecteur de l'avoir bien présente à l'esprit » que Kierkegaard, au tout début de son analyse, prend la peine de rappeler en quelques mots « le fameux avant-propos aux Sermons " ${ }^{15}$. Deux affirmations d'Adler retiennent particulièrement son attention. Dans la première, Adler, solennellement, dit " avoir eu une révélation par laquelle le Sauveur lui a communiqué une doctrine ${ }^{16}$ nouvelle. Cette affirmation, note Kierkegaard, est d'autant plus catégorique que le magister a pris soin de préciser que « le Sauveur lui ordonna de se lever, la nuit, et d'aller noter les mots suivants $»$. Dans la seconde, Adler, tout aussi irrévocable, raconte d'abord que c'est Jésus lui-même qui lui ordonna de brûler ses propres écrits (hégéliens) et de s'en tenir à la Bible; puis il ajoute que ses Sermons et ses Discours, depuis le no VI jusqu'à la fin, ont été écrits avec la grâce concomitante de Jésus, en sorte qu'il n'a été qu'un instrument. L'essentiel, pour Kierkegaard, est donc qu'Adler affirme que son élection par voie de révélation est analogue à l'élection d'un apôtre, et que, se disant l'instrument de la volonté divine, sa situation soit analogue à l'état d'inspiré ${ }^{18}$. Ainsi l'on retiendra surtout qu'Adler aurait non seulement eu une révélation au cours de laquelle il a reçu une doctrine directement du Sauveur, mais qu'il aurait écrit, en plus, tous ses Discours avec la grâce concomitante de Jésus. Reste à savoir si cette position initiale, si ce premier type de déclarations ne se trouvera pas altéré par les propos qu'il tiendra par la suite.

b) Le second type de déclarations considéré par Kierkegaard est celui qui fut fait en réponse aux questions posées en

Kongelige Bibliotek, Københovn), 1967. On y retrouvera sous la lettre U les livres non consignés dans le catalogue et ayant appartenu pourtant à Kierkegaard, dont : Quelques sermons, U 9, Documents concernant...., U 10, Quelques poèmes, H 1502, Essai de bref exposé ..., U 13, Études et exemples, U 11, ex Études théologiques, U 12.

15. Livre sur Adler, O.C., XII, p. 94. Voir notre note, no 12.

16. Ibrd., p. 95. Kierkegaard cite Adler.

17. Ibid., p. 95 .

18. Ibid., p. 96 . 
avril 1845 par la commission d'enquête constituée par les autorités ecclésiastiques ${ }^{19}$. Avec lui s'ouvre pour de bon le chapitre sur ce que Kierkegaard appelle volontiers l'«altération de la position fondamentale d'Adler par Adler lui-même ${ }^{20}$. C'est en particulier dans ses réponses à la deuxième question des autorités (question qui va droit au fait en lui demandant de reconnaître s'il a réellement eu une révélation, s'il le pense effectivement lui-même, etc. $)^{21}$, qu'Adler, pour la première fois, altère sa position fondamentale. L'un des premiers points de sa première réponse à la deuxième question des autorités est ainsi libellé : " Mais l'on peut être sauvé de façon miraculeuse, comme celle que j'ai décrite dans l'avant-propos des Sermons, c'est pour moi un fait que je ne saurais nier ${ }^{22}$. On y voit immédiatement qu'Adler ne donne pas suite à la question. On lui demandait, en somme, d'apporter une explication précise à cette histoire de révélation, non de parler d'autre chose. Car, de deux choses l'une, ou il aurait dû s'en tenir à sa position initiale ou il aurait dû la révoquer. Or, remarque Kierkegaard, Adler ne fait ni l'un ni l'autre :

Il ne maintient pas irrévocablement sa première déclaration, il la transforme ; et pourtant il veut faire passer ce changement pour une explication. Il ne s'en tient pas aux déclarations faites dans l'avant-propos à son propre sujet (et mises en cause par les Autorités) : nous le remarquons sans peine grâce à la différence qualitative fondamentale entre d'une part le fait d'avoir reçu une révélation et s'être vu confier une doctrine par le Sauveur et, d'autre part, le fait d'être sauvé de façon miraculeuse ${ }^{23}$.

Donc il est clair qu'Adler altère sa position initiale, dès le moment où il transforme le fait de sa révélation, au cours duquel il a reçu une doctrine nouvelle, en un simple éveil religieux qui lui apporte le salut ${ }^{24}$. Néanmoins, et parce qu'il ne voit pas la

19. La chancellerie avait en effet posé ses questions le 29 avril 1845 ; la réponse d'Adler s'était faite attendre jusqu'au 10 mai. De toutes ces questions et réponses nous n'avons retenu que les principaux points des réponses où Adler altère profondément son premier type de déclarations.

20. Livre sur Adler, O.C., XII, p. 93.

21. "La question 1) des Autorités est beaucoup moins importante, écrit Kierkegaard, et si je me suis attardé à la réponse d'Adler, c'est uniquement pour donner un avant-goût de la confusion où il se trouve ». Ibid., p. 103.

22. Ibid., p. 108.

23. Ibid., p. 109

24. Ibid., p. 103 
différence qualitative qui existe entre ces deux états, Adler ne fait pas pour autant mine de se rétracter. Au contraire, il veut faire passer cette transformation des données pour une explication qui lui permettrait de conserver tout le prestige d'un homme extraordinaire, pour ne pas dire d'un apôtre. C'est comme si, tandis qu'il s'était d'abord donné pour roi, il se donnait ensuite pour conseiller, pensant sérieusement que c'est du pareil au même. Mais Adler ignore tout à fait qu'un homme qui est ainsi sauvé de façon miraculeuse ne peut se trouver en même temps le dépositaire d'une doctrine nouvelle ${ }^{25}$; la conscience d'avoir été sauvé miraculeusement ne lui permettrait pas d'être cet homme exceptionnel ayant pour mission de transmettre un enseignement nouveau, elle l'humilierait plutôt, et le réduirait au silence, en lui rappelant constamment qu'il s'est égaré si loin sur le chemin de la perdition que seul un miracle pouvait le sauver $^{26}$. Aussi est-ce avec raison que Kierkegaard conclut que la première réponse d'Adler à la question 2) des autorités est celle d'un sophiste ou d'un étourdi ${ }^{27}$. En somme, si Adler a seulement été sauvé de façon miraculeuse, "suivant la conception propre et authentique qu'il se fait de lui-même, il n'a rien de nouveau à nous transmettre, aucune doctrine nouvelle, il n'en a jamais eu ${ }^{28}$. Adler devrait par conséquent révoquer solennellement sa première déclaration.

Toutefois Adler commence seulement de trahir sa position fondamentale. Répondant toujours à la question 2) des autorités, il ajoute : « Même si l'on ne voit dans mes Sermons et Études que les premiers balbutiements, la voix hésitante et faible d'un enfant, la Parole, je le crois, m'est pourtant témoin qu'un événement a eu lieu au cours duquel j'ai été saisi par la foi $"{ }^{29}$. Adler voudrait donc à présent faire preuve de modestie, mais d'une modestie, note Kierkegaard, qui est peut-être bien mal placée. Si Adler, en effet, prétend avoir noté les paroles du Sauveur en personne, avoir écrit tous ses Sermons sous sa dictée, comment peut-il alors comparer ces paroles aux balbutiements

25. Ibid., p. 110

26. Ibid., p. 110 .

27. Ibid., p. 114

28. Ibid., p. 115 .

29. Ibid., p. 110 . 
timides d'un enfant ${ }^{30}$ ? Car s'il est vrai, ainsi qu'il l'a déclaré dans son avant-propos des Sermons, que tout a été écrit avec la grâce concomitante de Jésus et qu'il n'a donc été qu'un instrument de la volonté divine, cette réponse devient absolument inadmissible. Elle ne saurait même être recevable, de renchérir Kierkegaard, que si l'avant-propos était tout entier le fruit d'un esprit troublé, auquel cas cette tentative littéraire pour présenter la révélation serait alors comparable aux explications hésitantes d'un enfant ${ }^{31}$. Donc, non seulement Adler substitue au fait d'être appelé par une révélation et d'être dépositaire d'une doctrine nouvelle celui d'avoir été sauvé de manière miraculeuse, - ce qui constitue une altération profonde à ses premières affirmations solennelles, mais en plus il compare les paroles reçues du Sauveur en personne à la voix hésitante et faible d'un enfant ; tout cela, évidemment, sans rien révoquer.

Cependant Aḍler ne s'arrête pas là. Il espère par ailleurs qu' « en élaborant plus longuement et en creusant ces idées à tête reposée, je me verrais désormais en mesure d'en développer le contenu chrétien sous une forme plus adéquate et mieux adaptée à la Parcie de la Sainte Écriture ${ }^{32}$. Chose certaine, pour un homme qui a reçu une révélation et qui a écrit sous la dictée du Sauveur, un tel espoir de perfectibilité ne peut être qu'accessoire $^{33}$. Comme le souligne justement Kierkegaard, Adler aurait plutôt dû craindre " que la longueur du temps ne ternisse la vivacité de la première impression; mais s'il a le front de mettre son principal espoir dans cette perfectibilité, il blasphème ${ }^{34}$. Adler ne peut donc laisser sous-entendre que les mots dictés par le Sauveur ne sont pas parfaitement adéquats, il ne peut pas dire que les paroles du Sauveur sont perfectibles, à moins, évidemment, d'avoir écrit et publié ces Sermons luimême, dans un réel état d'exaltation et de trouble, ou d'avoir complètement oublié l'inoubliable, c'est-à-dire cette révélation qu'il a eue au cours de laquelle le Sauveur lui a dicté une doctrine nouvelle, fait qư'il a solennellement affirmé. Or Adler ne pouvait 
pas oublier un tel fait ; il a donc écrit et publié ses Sermons luimême, sans le concours du Christ, et dans un état évident d'exaltation. De toute manière, à lui seul « cet espoir de perfectibilité implique essentiellement la révocation de l'avant-propos et des Sermons, car il fait des deux son œuvre personnelle ${ }^{35}$.

c) Nous passons à présent aux déclarations faites dans la dernière lettre datée du 5 juillet 1845 qui contient des éclaircissements sur les réponses qu'Adler a déjà apportées à la question 2) des autorités sur sa présumée révélation. Remarquons, avec Kierkegaard, que cette dernière lettre représente aux yeux d'Adler " la plus large concession possible ${ }^{36}$. Elle est conçue en ces termes :

Je ne prétends pas considérer mes Sermons (ou mes Études) comme des révélations parallèles au christianisme ou y faisant face, mais je considère les mots notés dans l'avant-propos des Sermons ainsi que des catégories dogmatiques dont j'use fréquemment, comme des points de repère indispensables à mon exaltation naissante pour conserver une forme au contenu chrétien ${ }^{37}$.

Aussi bien cette "plus large concession possible » revient-elle à dire qu'il n'y a pas de différence essentielle entre les mots écrits dans l'avant-propos et ses propres catégories dogmatiques, puisque les deux sont, en réalité, comme des " points de repère ", et font donc autorité au même titre. "Pourtant, remarque sévèrement Kierkegaard, les premiers sont écrits sous la dictée du Sauveur tandis que les secondes sont inventées par Adler ${ }^{38}$. Mais celui-ci n'y voit aucune différence qualitative : pour lui, les paroles de l'avant-propos (dont il est à présent certain qu'il a oublié qu'elles furent écrites sous la dictée du Sauveur) sont, en tant que points de repère, à mettre sur le même plan que ses propres catégories dogmatiques. De plus, les «points de repère " en question n'ont été indispensables qu'à son "exaltation naissante » (expression qui, en elle-même, constitue une sorte d'aveu à propos de ce qui s'est réellement passé la nuit de décembre 1842$)^{39}$, et seulement pour qu'il puisse conserver le

35. Ibid., p. 113 .

36. Ibid., p. 115 .

37. Ibid., p. 116.

38. Ibid., p. 118.

39. Ibid., p. 118. 
« contenu chrétien ». On pourrait, à juste titre, se demander comment ce qui était à l'origine une "nouvelle doctrine reçue du Sauveur » est devenu tout à coup ce qui permet de «conserver une forme au contenu chrétien ». Adler confondrait-il maintenant deux choses différentes ? - À moins qu'il ne se soit pas rendu compte de cette étourderie, ce qui, pour Kierkegaard, serait une autre preuve de l'état de confusion où il se trouve $e^{40}$. Finalement, Adler n'a rien apporté de nouveau, aucune doctrine nouvelle dont il aurait reçu la révélation, seulement des points dits de repère, indispensables à son exaltation naissante. ${ }^{41}$

Somme toute, pour Adler, les faits a) d'être appelé par une révélation et d'avoir reçu une doctrine du Sauveur, b) d'être sauvé de façon miraculeuse, c) d'avoir connu un moment d'exaltation sont presque identiques. Pourtant il ne s'agit pas, à l'intérieur de ces trois types de déclarations, de trois moments différents de sa vie, mais bien de ce moment décrit avec précision dans l'avant-propos des Sermons et présenté par la suite sous divers aspects. "Mais n'importe quel dialecticien, dit Kierkegaard, voit le dilemme : ou bien la réponse d'Adler est un non-sens, ou bien il lui faut révoquer solennellement sa première déclaration ${ }^{42}$. Or non seulement sa première déclaration est un nonsens, mais en plus Adler ne désavoue rien. Sans doute, "il est peut-être excusable du fait qu'il ne remarque réellement rien lui-même : dans cette mesure les motifs de l'excuse et de l'accusation portées contre lui sont identiques et imputables à la confusion où il se trouve ${ }^{43}$. De cette manière, personne n'aura conclu « directement » à l'égarement d'Adler ; seul Adler aura contribué à s'accuser lui-même, "indirectement ", en altérant à plusieurs reprises sa propre position fondamentale.

d) Non content d'avoir examiné si scrupuleusement les documents relatifs à la suspension et à la déposition du Magister Adler et d'en avoir tiré d'aussi évidentes conclusions, Kierkegaard s'est également attardé à l'analyse de ses quatre derniers

40. Ibid., p. 119.

41. Ibid., p. 120.

42. Ibid., p. 121.

43. Ibid., p. 121. 
écrits ${ }^{44}$. Dans les versions 2 et 3 du Livre sur Adler, on peut lire un texte d'introduction à l'étude de ces quatre ouvrages intitulé "Méthode suivie " ${ }^{45}$. Le texte, qui présente à nouveau le cas Adler, interroge, au fond, la raison d'être de ces quatre écrits, en posant le problème de leur nécessité dans les limites de la même méthode : nous venons de voir qu'Adler ne se comprend pas lui-même, qu'il n'est pas en accord avec lui-même, qu'il n'a pas une conception cohérente et quantitativement immuable de ce qu'est une révélation et qu'il ne s'en tient pas davantage avec une fermeté inébranlable à son assertion. Or Adler veut à présent être un auteur ; et nous sommes en droit d'exiger de lui qu'il nous donne d'abord une preuve manifeste qu'il s'est enfin rendu sérieusement compte de ce qu'il faut entendre par " révélation " au sens chrétien, et ensuite qu'il nous dise s'il se comprend maintenant dans son allégation initiale, quitte à la révoquer, ou à la réaffirmer solennellement pour pouvoir aller de l'avant, revêtu de ce caractère ${ }^{46}$.

Pourtant Adler, dans ses quatre derniers écrits, ne semble pas vouloir montrer qu'il est parvenu à se faire une conception cohérente de ce qu'il faut entendre par " révélation ", pas plus qu'il ne veut révoquer sa position fondamentale. Comme on va le voir, si "Adler semble maintenant vouloir faire des progrès et devenir - ou se contenter d'être - un génie ", par contre, " sa confusion fondamentale tient à ce qu'il fait néanmoins comme si de rien n'était et se croit en plein accord avec sa première déclaration (selon laquelle il était l'homme appelé par une révélation et à qui une doctrine était confiée) ${ }^{47}$. N'est-il pas étrange, aussi, qu'Adler ait jugé nécessaire de publier quatre livres d'un coup, comme s'il était pressé de se faire reconnaître ou comme s'il avait voulu faire bonne impression? S'il fallait donner la caractéristique principale de ces quatre ouvrages, il faudrait rappeler ce vers d'Horace que Kierkegaard tire des $\operatorname{Odes}(\mathrm{I}, 22,10)$ :

44. Ibid., p. 125. I1 s'agit des quatre ouvrages publiés d'un coup par Adler en 1846 : Études et exemples, Essai pour un bref exposé systématique du christianisme considéré au point de vue logique, Études théologiques, Quelques poèmes. Nous n'entrerons pas toutefois dans le détail de cette analyse, nous contentant de relever les remarques essentielles à l'établissement des preuves de la confusion d'Adler.

45. Ibid., p. 125, renvoi no 247

46. Ibid., p. 125 , renvoi no 247

47. Ibid., p. 125 . 
"Pendant que je chante ma Lalagé et que, insouciant, je vais à l'aventure plus loin que d'habitude ${ }^{48}$, dans lequel on reconnaît l'attitude d'un Adler insouciant, affirmant à travers tous ses écrits plus qu'il n'est ou ne peut comprendre. La Lalagé en question est représentative de la doctrine qui aurait été supposément transmise à Adler et dont il ferait maintenant l'exégèse dans ces livres, en la copiant le plus souvent, tout en ayant complètement oublié que cette doctrine lui a été communiquée par une révélation ${ }^{49}$. Oublier sa révélation par inadvertance, comme on oublie son chapeau ou comme les gens distraits peuvent oublier leur tête, n'est-ce pas pour le moins étrange ? Puis se mettre à bavarder, sans s'engager à fond, en cherchant, en trouvant et en ne trouvant pas ; vraiment cet homme ne peut avoir été appelé par une révélation ${ }^{50}$. Et pour Kierkegaard, en effet, si ces quatre derniers ouvrages ne contiennent plus « aucune allusion ni au fait de révélation ni à une révélation suivie ou à tel ou tel passage écrit sous l'impulsion directe de l'Esprit $"{ }^{51}$ et qu'ils « insistent, en revanche, et presque à vous en dégoûter, sur le génie " ${ }^{52}$, c'est que le Magister Adler, maintenant retiré dans son île de Bornholm, a l'esprit totalement dérangé. Car " débuter par une révélation pour conclure que, tout compte fait, l'on se qualifie par là de génie, comme on dit, vraiment, c'est le comble de l'étourderie et de la confusion ${ }^{53}$ !

Si donc, en guise de conclusion à cette longue introduction, il faut enfin résumer l'analyse de tous ces types de déclarations du Magister Adler, il convient de remarquer avant tout avec quelle efficacité cette application dialectique de la méthode indirecte nous a procuré ces quelques preuves de sa confusion. Adler seulement a contribué à accuser Adler en altérant, à trois reprises au moins, sa position fondamentale. L'étude des documents concernant sa suspension et sa déposition et celle de ses quatre derniers écrits a démontré, en effet, qu'Adler ne faisait aucune

48. Kierkegaard donnait ce vers en latin : " Dum meam canto Lalagen et ultra terminum vagor curis expeditis ». La traduction est donnée à la p. 130, renvoi 264.

49. Ibid., p. 131.

50. Ibid., p. 131.

51. Ibid., p. 132.

52. Ibid., p. 133. Kierkegaard a souligné à maints endroits de l'exemplaire Études et exemples qu'il possédait les passages où le mot " génie " apparaissait à tout instant.

53. Ibid., p. 134. 
distinction qualitative essentielle entre les faits a) d'être appelé par une révélation et d'avoir reçu une doctrine " directement " du Sauveur, b) d'être sauvé de façon miraculeuse, c) d'avoir connu un simple moment d'exaltation, et d) d'être finalement une sorte de génie commentant d'une manière à peine exégétique le texte d'une doctrine qui lui aurait été révélée. Ainsi quiconque aura le moindrement le sens du christianisme comprendra que les preuves de la confusion d'Adler sont dorénavant bien établies et qu'on peut affirmer à bon droit maintenant, en réponse à notre question de départ, que Dieu n'a jamais parlé à Adler, ni a fortiori ne lui a confié l'enseignement d'une doctrine nouvelle. Ces preuves de confusion sont même multipliées si l'on remarque qu'Adler a continué de croire jusqu'à la fin ce qu'il disait, ne révoquant rien de ses premières déclarations, se croyant conséquent sur toute la ligne . . . On serait alors porté à se demander comment il a pu sombrer si profondément dans une telle étourderie, - c'est ce que nous allons tenter de voir à l'instant, en faisant une analyse plus approfondie des causes qui expliquent cette confusion.

D'ores et déjà l'on peut dire que la confusion d'Adler trouve son explication dans trois principales " causes » ou " raisons ", qu'on peut énumérer dans cet ordre : 1) un choc émotif (ayant provoqué une confusion du subjectif et de l'objectif au plan psychologique), 2) un manque certain de formation conceptuelle chrétienne (ayant provoqué une confusion entre les concepts d' « apôtre " et de «génie ", de « révélation » et d'« exaltation »), 3) une mauvaise influence de la philosophie hégélienne (ayant provoqué une confusion du subjectif et de l'objectif au plan philosophique). Il faut dire de suite que Kierkegaard traite ces causes d'une manière un peu différente, parfois les entremêlant, d'autres fois les prenant plutôt une à une. Pour nous, la difficulté a surtout consisté à les présenter à la suite, de façon ordonnée et systématique, tout en respectant la méthode d'application de la dialectique. Si sans doute cette façon de faire ne peut redonner dans toute sa richesse le développement original, elle a du moins l'avantage de satisfaire à un désir tout naturel de cohérence en livrant en trois chapitres la simplicité d'une triple explication. 
1. PREMIÈre CAUSE : CONFUSION PRODUITE À LA SUITE D'UN CHOC ÉMOTIF - OU - CONFUSION DU SUBJECTIF ET DE L'OBJECTIF AU PLAN PSYCHOLOGIQUE

Le chapitre IV du Livre sur Adler s'ouvre sur un long exposé qui a pour but d'établir les principales raisons permettant de motiver sur le plan psychologique ce que Kierkegaard appelle la " catastrophe ", c'est-à-dire le bouleversement et la confusion survenus dans la vie du Magister Adler ${ }^{54}$. À l'intérieur de cet exposé, Kierkegaard explique notamment que la confusion d'Adler s'est produite à la suite d'un simple " choc émotif " 55 se référant à une notion aussi abstraite que : " être saisi par quelque chose de plus élevé, d'éternel, par une idée " ${ }^{56}$. Et, certes, il est vrai que toute religiosité relève jusqu'à un certain point d'un bouleversement intérieur de cette nature, d'une pression qualitative exercée sur le ressort de la subjectivité ${ }^{57}$. C'est pourquoi l'on peut dire que le mérite essentiel du Magister Adler, lequel à la différence de ses contemporains est totalement possédé par la religiosité au point qu'il se trouve, selon l'expression si chère à Kierkegaard, à « 70,000 brasses de fond ${ }^{58}$, tient effectivement dans le fait d'être saisi par une émotion religieuse de ce genre. Mais parce que l'intériorité du Magister relève dans ce cas du "domaine propre de l'homme ", de "l'homme pur et simple " $"$, il faut entendre ce bouleversement au sens d' " un choc qui ne fait pas de lui un autre homme, mais le réveille seulement et le fait revenir à lui-même ${ }^{60}$. À cet égard, l'émotion religieuse qui le saisit et les déterminations conceptuelles qu'il utilise pour parler de ce choc sont exclusivement humaines. Ainsi que Kierkegaard le fait remarquer, "l'on ne devient pas chrétien parce que l'on éprouve une émotion religieuse devant ce qui nous dépasse, et toute manifestation de ce genre n'est pas forcément chrétienne $"{ }^{61}$. Une émotion proprement chrétienne est en effet toujours contrôlée par des déter-

54. Ibid., p. 175 .

55. Ibid., p. 198.

56. Ibid., p. 198.

57. Ibid., p. 189.

58. Ibid., p. 192.

59. Ibid., p. 198.

60. Ibid., p. 199.

61. Ibid., p. 198. 
minations conceptuelles chrétiennes relevant de la sphère de la transcendance, tandis qu'une émotion tout court, une émotion au sens universel, qui naît d'un simple saisissement devant ce qui dépasse, donne plutôt cours à des déterminations païennes relevant de la sphère de l'immanence ${ }^{62}$. Pour s'exprimer en véritable chrétien, dit Kierkegaard, il faut, outre le langage du cœur, c'est-à-dire outre les déterminations conceptuelles nées de l'émotion simplement humaine, posséder aussi une connaissance pratique des déterminations conceptuelles chrétiennes ${ }^{63}$. Or la confusion d'Adler tient à ce que, n'ayant aucune connaissance stricte du langage conceptuel chrétien et se trouvant littéralement emporté par l' «impetus " (l'élan) de son émotion ${ }^{64}$, il a voulu exprimer coûte que coûte son état de choc. N'ayant alors aucun critère qualitatif auquel se référer pour savoir si son émotion est bien un réveil religieux chrétien, Adler, en tentant d'extérioriser son émotion, a sombré dans la confusion ${ }^{65}$. En conséquence, la catastrophe tient à ce qu'Adler, manquant de formation conceptuelle chrétienne et tout emporté par l'impetus de son émotion, se met à confondre le subjectif et l'objectif, c'est-à-dire à prendre le changement subjectif survenu en lui pour un événement extérieur ou « le fait d'avoir eu les yeux dessillés pour une révélation ${ }^{66}$. Aussi identifie-t-il son « réveil religieux ", ou si l'on veut « le fait d'être sauvé de façon miraculeuse » au fait de recevoir réellement une doctrine. Ce que madame Viallaneix exprime en d'autres mots : "Il confond, au mépris de leur radicale différence qualitative, ce qui vient de Dieu et ce qui appartient à l'homme. Il mélange, plus précisément, la Vérité révélée avec ses propres expériences " ${ }^{67}$. Adler est alors sujet à une exaltation sans frein ; il finit par se croire vraiment apôtre.

62. Ibid., p. 199. Kierkegaard définit l'immanence comme la sphère qualitative des relations des hommes entre eux en tant qu'hommes, dans laquelle tout acte humain ou naturel s'efface radicalement dans l'essentielle égalité de l'éternité. La transcendance est, quant à elle, une sphère qualitative à part, contenant tout ce qui vient objectivement de Dieu, avec lequel aucune égalité n'est pensable. Voir p. 136 et sv.

63. Ibid., p. 199.

64. Ibid., p. 208.

65. Ibid., p. 201.

66. Ibid., p. 202.

67. Viallaneix, Nelly. "Kierkegaard, prophète de la modernité ". Article paru dans le Bulletin de la Société de l'bistoire du Protestantisme français, tome cxxvii, juillet-août-septembre 1981, Paris, p. 371. 
Dès lors, la catastrophe psychologique du Magister Adler trouve son explication. Sa confusion prend son origine dans un choc, une sorte de secousse qui a provoqué chez lui un changement subjectif, à savoir une émotion religieuse relevant de l'humain (immanente), qu'il a prise pour un événement objectif relevant de Dieu (transcendant), parce qu'il n'avait pas une connaissance suffisante du langage conceptuel chrétien et qu'en dehors de ce cadre il s'est trouvé ballotté par ses sentiments.

\section{DEUXIÈME CAUSE : CONFUSION PRODUITE PAR UN MANQUE DE FORMATION CONCEPTUELLE CHRÉTIENNE}

Psychologiquement parlant, Adler aurait donc confondu un événement subjectif avec un événement objectif, une simple émotion avec une véritable révélation. Ainsi que nous l'avons déjà souligné, cette confusion s'explique pour une bonne part par un manque de formation conceptuelle chrétienne. Pour parvenir à cette conclusion, Kierkegaard a été contraint d'établir un critère permettant de distinguer la Révélation chrétienne des propos d'un illuminé. Ce sont les Écritures qui lui ont donc fourni le paradigme auquel il a pu comparer l'exaltation du Magister Adler : fondé sur l'activité du Christ et sur l'autorité des apôtres ${ }^{68}$, ce savoir conceptuel chrétien a permis à Kierkegaard de montrer qu'Adler n'était qu'un " faux Extraordinaire ", ignorant de ce qu'est une réelle " autorité divine ", et par conséquent incapable de faire la différence entre le comportement d'un « génie » et celui d'un « apôtre ». C'est en tout cas l'une des principales conclusions auxquelles il parvient dans Le livre sur Adler où il analyse en profondeur plusieurs concepts dogmatiques, tels les concepts d' « autorité divine » et de « révé-

68. Papirer I A 27, dans Soren Kierkegaard's Journals and Papers, édition de H.V. Hong, vol. 1, A-E, p. 168, no 412. Notre traduction : "Il me semble que la dogmatique chrétienne doit ressortir de l'activité du Christ, et a fortiori parce que le Christ n'a établi aucune doctrine ; il a agi. Il n'a pas enseigné qu'il y avait une rédemption des hommes, mais il sauvait les hommes. De l'enseignement de Mohammed a surgi une dogmatique musulmane (sit venia Verbo) mais c'est de l'activité du Christ qu'a surgi la dogmatique chrétienne. La nature du Christ fut donnée à travers son activité comme Christ (ce qui, en fait, était le principal de la chose). La relation du Christ à Dieu, à l'homme, à la nature, et la situation humaine furent qualifiées par son activité. Tout le reste doit être considéré seulement comme un préambule. 5 novembre 1834 ». 
lation ${ }^{69}$, et qu'il s'est confirmée à lui-même à la suite de la rédaction, en 1847, d'un article important Sur la différence entre un génie et un apôtre, qu'il fit paraître, en 1849, dans ses Deux petits traités éthico-religieux.

$\mathrm{Si}$, dans ses quatre derniers écrits, Adler semble se contenter d'être un " génie ", sa confusion fondamentale tient toujours à ce qu'il croit être en accord avec son premier type de déclarations selon lequel il aurait bel et bien reçu une révélation qui ferait de lui un « apôtre ». Aussi Kierkegaard consacre-til plusieurs pages à l'éclaircissement de ces deux déterminations conceptuelles. Le livre sur Adler nous apprend que a) le génie et l'apôtre se distinguent qualitativement en ce sens qu'ils appartiennent chacun à une sphère qualitative. La détermination " génie » relève de la sphère de l'immanence tandis que la détermination "apôtre " relève de la sphère de la transcendance ${ }^{70}$. "Tout acte de penser s'exerce dans l'immanence ; en revanche, le paradoxe et la foi constituent une sphère qualitative à part $"{ }^{71}$. Tout acte de penser s'exerce dans l'immanence parce qu'il a cours dans " les relations des hommes entre eux en tant qu'hommes" et "dans l'essentielle égalité de l'éternité " ${ }^{72}$. En ce sens le génie relève de l'immanence parce que la nouveauté qu'il apporte, toute différente qu'elle puisse paraître à son époque, finit un jour ou l'autre par être assimilée à l'espèce, le condamnant en définitive à disparaître. Par conséquent même la "différence " du génie s'évanouit devant l'essence de la pensée éternelle. De plus, le " génie, comme le mot l'indique (ingenium, de naissance ; primitivité, primus ; originalité, origo ; spontanéité, etc.) est l'immédiateté, une donnée naturelle; il est inné ». Le génie possède naturellement toutes ses rares facultés même s'il ne se tourne pas vers Dieu. Il en va tout autrement de l'apôtre

69. "In his first Preface Kierkegaard says he uses the Adler case" to defend dogmatic concepts", and in the second Preface he claims that from the book one will "get clarity about certain dogmatic concepts and an ability to use them". Remarque de Stankey Cavell dans son article "Kierkegaard's On Authority and Revelation" in Kierkegaard : A Collection of Critical Essays, edited by Josiah Thompson. Garden City, New York, Anchor Books, Doubleday \& Company inc., 1972 (Modern Studies in Philosophy), p. 377. La première préface est donnée dans Paptrer VIII ${ }^{2}$ B 270, p. 309 ; la deuxième dans Papirer VIII $^{2}$ B 27, p. 75.

70. Livre sur Adler, O.C., XII, p. 136.

71. Ibid., p. 137. Le paradoxe religieux appartient à la sphère transcendante parce qu'il repose sur la différence qualitative essentielle et éternelle qui distingue Dieu de l'homme.

72. Ibid., cette citation et les suivantes sont tirées de la p. 137 . 
qui, lui, relève de la transcendance. « Le mot lui-même marque la différence ; on ne naît pas apôtre ". Il n'est nullement question ici d'une donnée naturelle ou d'une égalité partagée avec les hommes. La différence qualitative essentielle, c'est que "l'apôtre est un homme appelé par Dieu et qui reçoit de lui une mission à remplir ". Sa vocation " est un fait-paradoxe qui, à la fin comme au début de sa vie demeure tel en dehors de son identité avec lui-même ". Cette vocation ne fait pas de lui un génie, elle ne lui donne pas un cerveau d'élite, ne lui obtient pas un degré supérieur, par exemple, d'imagination ou de perspicacité ; l'apôtre, au contraire, reste ce qu'il est, quoique ce fait-paradoxe fasse de lui un homme à jamais différent des autres hommes, un envoyé de Dieu chargé d'aller dans le monde proclamer la parole en dépit de tous les obstacles ${ }^{73}$.

Mais il est un autre point sur lequel Kierkegaard insiste particulièrement : «- b) Un génie est ce qu'il est par luimême, c'est-à-dire par ce qu'il est en lui-même ; un apôtre est ce qu'il est par son autorité divine $"{ }^{74}$. La doctrine apportée par le génie sera donc évaluée strictement au point de vue esthétique ou philosophique selon les règles qui ont cours dans la sphère immanente et en regard des qualités que l'homme possède en lui-même. Mais pour l'apôtre, c'est exactement l'inverse ; parce qu'il part en son argumentation du fait de la révélation et de l'autorité divine dont il est investi, je dois absolument m'incliner devant lui et me garder d'interroger la valeur esthétique ou philosophique de sa prédication ${ }^{75}$. "Je ne dois pas écouter Paul parce qu'il est un grand, un incomparable esprit, mais je dois

73. Ibid., p. 138. On peut lire sur la même page : « Ce fait-paradoxe rend à jamais l'apôtre par là même différent de tous les autres hommes. Le nouveau qu'il peut avoir à annoncer est essentiellement de l'ordre du paradoxe, et nulle immanence ne peut l'assimiler. L'apôtre ne se comporte pas comme l'homme exceptionnellement doué qui devance son temps ; il a peut-être été ce que nous appelons un simple, mais, par un fait-paradoxe, il a été appelé à annoncer son message. Et même si la raison croyait pouvoir s'assimiler la doctrine, la manière dont celle-ci est entrée dans le monde lui échappe ; car, en son essence, le paradoxe est précisément la protestation élevée contre l'immanence ; mais la manière dont la doctrine est entrée dans le monde est le facteur qualitatif décisif, et l'on ne saurait l'omettre que par fraude ou irréflexion ". Et dans son article Sur la différence entre un génie et un apôtre, il écrit : que l'apôtre est un " homme appelé par une révélation pour aller dans le monde proclamer la parole, agir et souffrir, pour consumer sa vie dans une incessante activité comme envoyé du Seigneur ", O.C., XVI, p. 160.

74. Livre sur Adler, O.C., XII, p. 136.

75. Ibid., cette citation et les suivantes sont tirées de la p. 138. 
m'incliner devant lui parce qu'il est revêtu de l'autorité divine ; et, que je le fasse ou non, il garde en tout cas la responsabilité de produire cette impression d'autorité ». En ce sens, "l'autorité divine est le facteur qualitatif décisif»; elle fonde la différence qualitative essentielle entre l'apôtre et le génie.

Mais il y a tout de même une complication : à quoi reconnaît-on cette autorité ? Dans l'ordre immanent, au moins, un sceau peut garantir qu'il s'agit effectivement de l'autorité royale, et c'est la forme et le fond du discours qui témoignent de la même façon de l'autorité du poète et du penseur ; - mais pour Dieu, qu'est-ce qui rend compte de son autorité ? Certes, répond Kierkegaard, " un roi appartient à ce monde sensible ; on peut s'en convaincre par les sens, et s'il le faut, il peut aussi à l'occasion le prouver. Mais Dieu ne se manifeste pas de la même manière, (. . .) il ne peut pas se mettre au service des hommes en leur apportant la certitude matérielle qu'un apôtre est un apôtre - ce serait un non-sens ${ }^{76}$; il ne peut pas nantir son envoyé de secours visibles à la manière d'un roi qui donne à son ambassadeur une escorte et un sceau que tous connaissent. Et même un miracle, si l'apôtre en a le don, ne peut procurer la certitude sensible de son autorité, puisque le miracle est un objet de foi. Et comme il y a « un non-sens à recevoir la certitude sensible de l'être de Dieu, qui est Esprit ", il y a aussi " un non-sens à recevoir la certitude sensible qu'un apôtre est un apôtre ». Aussi bien l'envoyé de Dieu et sa doctrine doivent-ils être acceptés, comme on dit, en quelque sorte a priori, parce que leur autorité relève d'une sphère qualitative à part, sans rapport au jeu de la pensée ${ }^{77}$. C'est pourquoi l'apôtre est jus-

76. Ibid., cette citation et les suivantes ... p. 140.

77. Dans son Journal (extraits, 1846-1849), Kierkegaard distingue encore les deux sortes d'autorité en ces termes : " L'autorité n'est ni d'être roi, ni d'être empereur, général, d'avoir la force des armes, d'être évêque ou encore agent de police (en marge : il s'agit ici du concept d'autorité dans l'immanence, et non pas dans le paradoxe) ; mais c'est, dans une ferme et constante résolution de vouloir sacrifier tout, même sa vie pour une cause, de plaider une cause tout en étant pleinement d'accord avec soi-même qu'on a besoin de rien, qu'on ne craint rien. Cette intransigeance de l'infinitude, voilà l'autorité (et en marge : C'est pourquoi celui qui a l'autorité s'adresse toujours à la conscience, non à l'intelligence, à la perspicacité, à la profondeur, bref, à l'homme et non au professeur). La véritable autorité, c'est quand votre cause est la vérité. Si les propos des Pharisiens étaient sans autorité, quoique leur enseignement fût bien celui des maîtres autorisés, c'est que leurs propos comme leur vie étaient dans les griffes temporelles de trente-six égards à ménager ". Journal, tome II, traduction de Knud Ferlov et Jean-J. Gateau. Paris, Librairie Gallimard, NRF, 1954, p. 171. VIII A 416. 
tement investi de l'autorité divine, pour tenir la doctrine à distance des impertinences et empêcher de la sorte qu'en raisonnant sur la forme et le fond de ses prédications les hommes ne le rabaissent au statut de simple candidat porteur d'une doctrine nouvelle ${ }^{78}$. L'autorité divine doit, en effet, être acceptée toute d'un bloc, précisément parce qu'elle est divine. C'est un fait éminemment compréhensible, dès qu'on a saisi que même l'intelligence la plus complète de la doctrine ne fait pas qu'on possède cette autoritét ${ }^{79}$.

Comme on le voit, toutes ces considérations autour du concept d'autorité viennent de ce qu'il s'agit de ne pas confondre deux autorités très différentes, appartenant chacune à une sphère qualitative spécifique. "Dans la sphère de l'immanence, poursuit Kierkegaard, l'autorité ne se prête en aucune façon au jeu de la pensée, ou elle ne s'y prête qu'à titre transitoire " ${ }^{80}$. En matière politique, civique ou sociale, par exemple, l'autorité invoquée ne représente en effet « qu'un moment transitoire appelé à disparaître soit à la longue dans le temps, soit avec la temporalité et la vie terrestre constituant elle-même un moment transitoire qui s'évanouit avec toutes les différences qu'il comporte ${ }^{81}$. En outre, nous avons vu qu'au fond de tout rapport des hommes entre eux en tant qu'hommes, il n'y a de différence concevable (du type de celle que représente à titre transitoire l'autorité d'un génie) qu'au sein de l'immanence ou de l'essentielle égalité des hommes entre eux. Autrement dit, la différence - ou l'autorité dans l'immanence, disparaît aussitôt qu'on la pense sous l'angle de l'éternité. Ainsi, " comme sujet, je dois honorer le roi et lui obéir de toute mon âme, mais il m'est permis de trouver mon édification religieuse dans la pensée que je suis en mon essence citoyen des cieux, et que, si j'y rencontre un jour feu le roi, je ne serai pas tenu envers lui à l'obéissance du sujet ". Par conséquent, si l'on parle de l'autorité dans la sphère de la transcendance, c'est-à-dire au point de vue apostolique, alors entre en jeu dans les rapports de Dieu aux hommes une différence qualitative essentielle qui marque

78. Ibid., p. 140.

79. Ibid, , p. 140.

80. Ibid., p. 141.

81. Ibid., cette citation et les suivantes . . . p. 142. 
une éternelle inégalité. C'est parce que cette différence est impossible à penser en termes d'égalité avec Dieu qu'elle est essentiellement paradoxale et qu'elle confere à l'apôtre cette qualité spécifique qui fait qu'il ne peut plus entrer dans le cadre des relations des hommes entre eux en tant qu'hommes, au point où son autorité, parce qu'elle est d'essence éternelle, prédomine sur toute autorité transitoire.

Mais, en tout ceci, l'apôtre peut-il trouver le moyen de montrer qu'il est essentiellement qualifié par Dieu pour jouer son rôle ? Autrement dit, comment, maintenant, peut-il prouver qu'il a autorité ${ }^{82}$ ? - Compte tenu du paradoxe religieux, " il n'a pas d'autre preuve que son affirmation. Et il faut qu'il en soit ainsi ; sinon le croyant serait avec lui en relation directe, et non dans le rapport du paradoxe ". C'est parce qu'il est ce qu'il est par l'hétérogénéité qu'il tient de ce paradoxe que l'apôtre doit garder l'autorité divine dont il est investi absolument inchangée. Du moins peut-on le reconnaître, en toute dernière analyse, à sa volonté de tout souffrir pour sa mission. Aussi l'obéissance inconditionnelle de l'apôtre à la volonté divine estelle la forme de son autorité. Car si, d'une part, l'apôtre use de son autorité et se réclame de Dieu, cette autorité, d'autre part, doit reposer en même temps sur son obéissance inconditionnelle.

Néanmoins cette obéissance inconditionnelle n'est pas le seul signe auquel on reconnaît l'apôtre véritable. À la différence du génie, qui n’a qu'une " téléologie immanente ", l'apôtre, lui, a une "téléologie absolue ». Au premier, la doctrine est donnée pour lui-même, comme pour être soumise à son examen, mais au second, elle est donnée exclusivement pour qu'il puisse enrichir les autres ${ }^{83}$. Et c'est ce qui différencie encore l'apôtre du génie, puisque le génie "vit en lui-même ", " ne se pose pas dans un rapport téléologique vis-à-vis du monde et des autres ", et qu' " aucune de ses œuvres n'a son telos (sa fin) audehors ${ }^{84}$, étant donné qu' «'il n'écrit pas afin de, afin. d'éclairer les hommes, afin de les aider sur la bonne voie $"{ }^{85}$, mais seulement pour se rapporter à lui-même.

82. Ibid., cette citation et les suivantes . . p. 147.

83. Deux petits traités éthico-religieux, O.C., XVI, p. 160.

84. Ibid., p. 161.

85. Ibid., p. 162. 
De plus, et parce que la Providence ne peut ignorer les attributs qui sont propres à son époque, l'apôtre véritable doit posséder la réflexion à un degré éminent ${ }^{86}$, premièrement, parce qu'il doit avoir la profondeur d'esprit suffisante pour « réaliser à fond, existentiellement, une idée en relation immédiate avec Dieu $"{ }^{87}$, et, secondement, parce qu'il doit " disposer de toute sa prudence réfléchie au moment où il s'introduit, lui et sa révélation, dans la continuité du temps ${ }^{88}$. À ces deux lieux de réflexion correspond, au point de vue éthico-religieux, une double responsabilité. La première, tout intérieure, le concerne personnellement, à cause de sa divine obligation et de la terrible responsabilité qui lui incombe à chaque pas : aussi l'apôtre véritable doit-il encore se demander constamment s'il fait bien ce qui lui a été demandé et si c'est bien la voix de Dieu qu'il a entendue $^{89}$. Cette incertitude, qui suscite en lui la crainte et le tremblement, exerce sur sa conscience une pression telle, qu'elle le maintient, en dépit de ses qualités immanentes, dans la plus totale humilité. Mais, paradoxalement, et parce que le véritable apôtre a aussi son assise extérieure dans son rapport absolu avec Dieu, il doit être, devant ce dernier et dans son rapport non équivoque avec les autres hommes, sûr qu'il se comprend luimême, dans le fait que cet événement extraordinaire lui est personnellement arrivé, et certain du contenu de sa révélation ${ }^{90}$. Quant à la seconde responsabilité, elle est, précisément en raison du salut suprême qu'il représente, tout extérieure, relative aux autres hommes en général et en particulier à l'ordre établi ${ }^{91}$. Dans l'exercice de sa téléologie absolue, l'apôtre véritable est tenu d'user pleinement de son autorité, parce qu'il est, expressément, appelé à se prévaloir d'elle, même s'il apporte avec lui le «scandale " qui le rend odieux à l'ordre établi. Si donc il n'a nul besoin de l'approbation de la majorité, c'est qu'il lui importe peu de triompher " aujourd'hui, demain ou dans mille ans, car il a déjà triomphé ; son rapport avec Dieu est sa vic-

86. Livre sur Adler, O.C., XII, p. 54.

87. Ibid., p. 57.

88. Ibid., p. 54

89. Ibid., p. 54.

90. Ibid., p. 51 et Annexes IV, p. 247.

91. Ibid., Annexes IV, p. 247. 
toire ${ }^{92}$; il ne vit plus désormais au plan des rapports des hommes entre eux en tant qu'hommes, leur souci du triomphe lui sera pour toujours étranger. Au reste, l'acte le plus lourd de signification que le véritable apôtre puisse commettre en vertu de sa responsabilité sociale, c'est encore de ne plus se soumettre à l'autorité de l'ordre établi, de ne plus la reproduire en luimême, et d'adopter, à l'encontre de cet ordre, une attitude réformatrice ${ }^{93}$.

En ce cas, et pour en revenir finalement à Adler lui-même, on comprend immédiatement que toutes ces explications autour des concepts d' "apôtre ", de "révélation " et d' " autorité " ne font que desservir sa cause, car il suffit de le comparer à un véritable apôtre pour faire la découverte du manque de rapport. En premier lieu, et de toute évidence, le Magister Adler n'a aucune connaissance de ce qu'est le concept d'autorité divine qui fonde la différence qualitative essentielle et éternelle permettant de distinguer l'apôtre du génie. De là qu'il ne peut faire état de quoi que ce soit qui, au point de vue chrétien, légitimerait sa condition d'apôtre. Quand, en effet, un homme est investi de l'autorité divine et qu'il se trouve dans la situation introduite par le paradoxe religieux, c'est afin de tenir la doctrine à l'écart des impertinences et de garder au contenu de son affirmation une allure inchangée. Mais il est impossible de penser avoir affaire à la prophétie homogène d'un élu lorsqu'on se trouve en face d'un discours aussi incohérent que celui qui altère à trois reprises au moins sa position initiale. Cette « étourderie dialectique " montre en tout cas qu'Adler, contrairement à un véritable apôtre, ne possède point un degré très éminent de réflexion ; un véritable apôtre n'aurait jamais pris un « simple réveil religieux " ou une " exaltation subjective » pour une grâce exceptionnelle. En plus, dans ses quatre derniers écrits, Adler prétend être un génie, donc dit avoir des qualités naturelles que l'apostolat, nous l'avons vu, ne procure pas. Ensuite l'attitude d'Adler devant la commission d'enquête ne laisse planer aucun doute quant à son statut authentique. Un véritable apôtre n'aurait seulement pas accepté de se soumettre à un tel examen, à cause

92. Ibid., p. 51.

93. Ibid., p. 40. 
du paradoxe religieux où il se trouve qui l'empêche d'être en relations directes avec les autres hommes et de traiter avec eux sur un plan d'égalité, d'autant plus que la différence éternelle à laquelle il est tenu prédomine sur toute autorité transitoire. En outre ses concessions montrent qu'il n'est pas prêt à tout supporter au nom de Dieu et qu'il ne consume pas sa vie au service de celui-ci. Et de quelle obéissance inconditionnelle, signe d'autorité apostolique, peut-on bien parler, quand un homme oublie jusqu'à sa révélation ? Et sans révélation, quelle position téléologique absolue peut encore être la sienne ? Ses quatre derniers ouvrages, dans lesquels il s'écoute parler, évoquant ici et là son « génie ", restent « sans nul telos pour personne, comme un poète s'intéresse aussi peu au lecteur qu'un rossignol à celui qui l'écoute ${ }^{94}$. Du reste, s'il s'agit uniquement d'un "réveil religieux », comme il l'a déjà prétendu, pourquoi adopter alors une position téléologique absolue ? « Le réveil religieux ne comporte pas la même exigence ; touché par lui de façon surnaturelle l'on n'est pas tenu de l'annoncer aux autres ${ }^{95}$.

Enfin, si l'on examine la chose sous l'angle de la responsabilité éthique intérieure, Adler ne supporte pas davantage la comparaison. La confusion à laquelle il s'abandonne ne permet guère de penser qu'il réalise à fond, existentiellement, son dit apostolat, pas plus qu'il ne se rend compte de l'immensité de la responsabilité qu'il a revendiquée. Lorsqu'il écrit, il va, sans le moindre scrupule, insouciant et génial, chantant sa Lalagé ; rien ne le maintient dans l'humilité, dans la crainte ou dans le tremblement. Sans doute une fois la crainte l'a-t-elle saisi, lors de son interrogatoire, mais c'était alors plus la crainte des autorités que des foudres de Dieu. Encore là, on voit bien qu'il n'avait pas son assise dans un rapport direct avec l'Absolu, puisqu'il n'était pas sûr de lui, qu'il ne se comprenait pas lui-même et, finalement, qu'il ne croyait pas avoir reçu une révélation. De même, si l'on considère Adler sous l'angle de la responsabilité éthique extérieure, on est immédiatement frappé par la façon dont il s'introduit à l'ordre établi. Plutôt que de quitter son

94. Livre sur Adler, O.C., XII, Annexes 2, la version 2 du livre, p. 242.

95. Ibid., Annexes 4, Autre rédaction du chapitre 1, p. 248. 
poste, comme le ferait n'importe quel apôtre convaincu qu'il apporte un point de départ nouveau, le Magister Adler, chose étonnante, réclame que cet ordre le garde à son service; ce qui est proprement une contradiction ${ }^{96}$. Donc, il n'adopte pas l'attitude réformatrice de l'apôtre puisqu'il réclame qu'on le soumette à une enquête. Au fond, en essayant à la fois de se concilier les faveurs des autorités (parce qu'il demande à être soumis à une commission d'enquête) et de conserver son statut d'apôtre (en ne révoquant pas pour autant le fait de sa prétendue révélation), Adler voudrait en quelque sorte que l'ordre établi l'authentifie dans son rôle : ce qui est tout à fait de la folie. Un véritable apôtre se serait depuis longtemps rendu odieux à l'ordre établi en se prévalant de son autorité divine, il aurait partout semé le doute et le scandale, surtout parmi la foule, dont il n'aurait pas mendié, à la manière d'Adler, les approbations enthousiastes, et personne n'aurait joyeusement signé de pétition en sa faveur. Mais quel acte lourd de signification Adler a-t-il accompli pour acquérir sa compétence d'apôtre dans un tel « discrimen ${ }^{97}$ ? Aucun, à la vérité.

Aussi la conclusion est-elle, en définitive, assez facile à tirer : Adler n'a jamais été un "apôtre », il n'a jamais jeté de différence qualitative essentielle et éternelle dans ses rapports avec les autres hommes, pas plus qu'il n'a eu de "révélation » ou n'a été investi d'une quelconque "autorité divine ". Simplement son impetus émotif, joint à un manque de savoir conceptuel chrétien, a fait qu'il n'a eu « aucun respect convenable pour la détermination qualitative de ces concepts ${ }^{98}$ et qu'il s'est mis, "bona fide ", à proférer des blasphèmes ${ }^{99}$ en les confondant avec ceux qui qualifient plutôt le génie. En fait Adler est toujours demeuré dans la sphère immanente. Aussi le problème qui se pose alors n'est-il plus d'expliquer, au point de vue de la transcendance, si le génie peut supporter la comparaison avec l'apôtre, mais de savoir pour quelles raisons, au point de vue philosophique, donc immanent, un génie peut finir par se prendre pour un apôtre.

96. libid., p. 32.

97. Ibid., p. 32.

98. Ibid., p. 173.

99. Ibid., p. 173. 
3. TROISIÈME CAUSE : CONFUSION PRODUITE PAR LA PHILOSOPHIE HÉGÉLIENNE - OU — CONFUSION DU SUBJECTIF ET DE L'OBJECTIF AU PLAN PHILOSOPHIQUE

Adler, au cours de sa vie, comme on l'a vu au tout début de notre exposé historique, s'est familiarisé avec la philosophie allemande, et en particulier avec le hégélianisme dont il n'a pas tardé à devenir un disciple convaincu. Or, parce qu'il a immédiatement compris que cette soi-disant révélation constituait un exemple parfait de confusion due à la philosophie hégélienne et que la situation du Magister était bien celle à laquelle conduisait inéluctablement cette philosophie dans ses implications théologiques, Kierkegaard a inclu, dans le plan de son ouvrage, toute une partie de chapitre consacrée à l'analyse d'Adler comme satire indirecte de l'hégélianisme ${ }^{100}$.

Adler, c'est un fait, est resté lié à la philosophie hégélienne. Rappelons simplement que, juste avant d'avoir sa « révélation ", il terminait d'écrire un cours sur la logique subjective de Hegel. En outre, le fait qu'il ait brûlé tous ces manuscrits hégéliens aussitôt après son extraordinaire expérience ne signifie nullement qu'il se soit débarrassé pour de bon de sa toute récente formation philosophique. Bien au contraire, puisque le sentiment qu'il a de caractériser très exactement et avec une précision toute philosophique sa propre transformation subjective par cette image de la "révélation » lui est directement inspiré par son instruction scientifique ${ }^{101}$. Car s'il y a confusion lorsqu'il qualifie de "révélation » son propre changement subjectif, c'est précisément parce que la philosophie hégélienne, à laquelle il demeure attaché, est impropre à contenir pour de vrai le concept qualitatif et paradoxal de révélation ${ }^{102}$. Pour Kierkegaard,

La philosophie de Hegel est proprement la philosophie de l'identité historicisante qui suit un processus universel en ce sens que l'individu particulier n'est pas à l'origine de tout développement, mais que l'humanité, en tant que subjectivité, comprend tous les moments de l'évolution dans l'identité immédiate du sujetobjet. Nulle part il n'y a de point de départ transcendant,

100. Ibid., Chapitre IV : "Conception psychologique d'Adler en tant que phénomène et comme satire de la philosophie hégélienne ... ", p. 175.

101. Ibid., p. 205.

102. Ibid., p. 207. 
immuable sur le plan qualitatif, tel qu'aux yeux de la conception inverse il s'incorpore au domaine global de la subjectivité (l'humanité). Tout point de départ nouveau devient dès l'instant suivant, un moment du processus et nous apparaît ainsi comme simple détermination de la subjectivité übergreifende (prépondérante), c'est-à-dire de l'humanité, de l'espèce humaine. Or le christianisme se pose comme point de départ transcendant, comme une révélation, en sorte que l'immanence est à jamais incapable de s'assimiler ce point de départ pour en faire un moment. (. . .) la philosophie hégélienne (. . .) invente la médiation et prive le concept de révélation de toute consistance. Elle ne nie pas la révélation, mais l'interprétation qu'elle en donne n'en laisse rien subsister ; elle explique tant et si bien le concept de révélation qu'à la fin ce dernier traduit le côté immédiat de la subjectivité, non pas individuelle, mais de celle propre à l'espèce humaine, à l'humanité. Et voici la méthode à flot, la philosophie de l'identité historicisante a remporté un triomphe absolu. ${ }^{103}$

C'est donc parce que le hégélianisme ne peut s'assimiler de paradoxe qualitatif d'ordre transcendant, tel qu'on le trouve dans le christianisme, que l'explication qu'Adler donne de sa révélation et de sa rupture avec cette philosophie paraît si confuse.

L'on voit aisément que la confusion vient de l'imprécision du concept de révélation, appelé à définir tantôt la rupture d'avec la philosophie hégélienne d'identité et le processus immanent, tantôt, dès qu'on l'examine de plus près, la simple transformation subjective précisément définie par le concept hégélien : une révélation. ${ }^{104}$

En outre, note Kierkegaard, "si l'on est formé, pour ne pas dire déformé, aux seuls principes de la dialectique hégélienne selon laquelle tout revient à peu près au même ", on peut abolir complètement toute distinction qualitative entre les concepts ; à tel point qu' " un dialecticien de cette sorte, vivant fort à l'aise dans sa chimère, admet sans peine que les faits a) d'être appelé par une révélation et d'avoir reçu une doctrine, b) d'être sauvé de façon miraculeuse, c) d'avoir connu un moment d'exaltation sont à peu près identiques ${ }^{105}$. C'est pourquoi il est si facile à Adler de concevoir comme ne faisant qu'un seul moment ce que nous pourrions prendre pour trois moments différents de sa vie,

103. Ibid., p. 205.

104. Ibid., p. 205.

105. Ibid., p. 115 . 
parce qu'il lui était aisé de « justifier ce qu'il avait pris pour une expérience révélatrice en y voyant un moment historique de l'Esprit du monde venu se focaliser pour un temps dans ses propres écrits " ${ }^{106}$. En somme, Adler était hégélien, « non seulement dans sa manière de concevoir la Révélation comme la suite ininterrompue de moments historiques dont il aurait été l'un des représentants, mais aussi dans la dialectique de justification qui dut l'animer " ${ }^{107}$. Dès lors, « avoir eu une révélation au cours de laquelle on a reçu une doctrine " ou « être sauvé de façon miraculeuse ", ou " avoir connu un moment d'exaltation " revient exactement au même, dans la mesure où la dialectique hégélienne peut médiatiser le paradoxe et ramener celui-ci au plan immanent. C'est la raison pour laquelle, dupe lui-même de la philosophie dont il pense s'être débarrassé, Adler passe progressivement de la sphère transcendante à la sphère immanente et finit par se présenter à la fois comme " apôtre » et comme "génie ». Mais dans un système où "la méthode historicisante a remplacé l'idée de fondement par celle de genèse ${ }^{108}$, le risque est forcément plus grand de glisser d'une détermination conceptuelle à une autre, sans égards pour leurs différences qualitatives. Ainsi, il y a chez Adler une confusion très nette entre la sphère éthico-philosophique ou métaphysique de l'immanence et la sphère éthico-religieuse de la transcendance. Compte tenu de ses explications dénuées de sens chrétien, et parce que le hégélianisme abolit littéralement toute distinction qualitative éternelle, on comprend qu'Adler a réduit son expérience religieuse à la sphère éthico-philosophique, donc à celle des relations et des spéculations des hommes entre eux en tant qu'hommes, où il espère trouver l'approbation du peuple et le consentement des autorités religieuses. Mais, tandis qu'il déprécie et médiatise sa prétendue vocation d'apôtre en rabaissant de la sorte le dialogue au niveau des intellectuels de la religion, Adler, simultanément, essaye de maintenir sa position initiale. Encore tout chargé de son bagage métaphysique, il maintient donc qu'il est apôtre, - sans voir toutefois que la sphère éthico-religieuse commande une pratique autrement

106. Ibid., Introduction de Jean Brun, p. xviii.

107. Ibid., Introduction de Jean Brun, p. xx.

108. Dialectique de la communication éthique et étbico-religieuse, O.C., XIV, p. 362, en note. 
sérieuse, sur le plan de l'existence, que toute cette façon hégélienne de concevoir comme moment ${ }^{109}$. Car si la formation scientifique du Magister Adler ajoute tellement à sa confusion, c'est parce que la médiation hégélienne l'a empêché de « vivre à fond » une intériorité éthico-religieuse qui exige en réalité une aspiration morale et une passion religieuse plus prononcées que celles, très fantaisistes, qui l'ont animé.

En ce sens, la dialectique kierkegaardienne, qui est existentielle, et qui, à ce titre, concerne avant tout l'expérience vécue et approfondie de la subjectivité, montre "l'impossibilité d'un système logique de l'existence, c'est-à-dire d'un système où l'existence est pensée plutôt que vécue ${ }^{110}$. En cela, elle s'oppose radicalement à la dialectique hégélienne, qui, elle, est développement à la fois rationnel et réel, logique et ontologique de la réalité, mais qui, selon Kierkegaard, demeure sans rapport avec la vie qu'elle se contente de reproduire. Ainsi peut-on comprendre, de façon générale, l'influence de la dialectique hégélienne sur le Magister Adler, dès qu'on s'aperçoit que sa vie et sa pensée résident dans le momentané ${ }^{111}$, lui donnant cette allure un peu désinvolte et abstraite qui, malheureusement, le fait passer au-dessus des sobres exigences de la sphère éthicoreligieuse. C'est alors que la dialectique kierkegaardienne, et jusque dans l'application présente, s'oppose à celle d'Adler, dans la mesure où justement, chez Kierkegaard, "c'est la notion d'intériorité éthico-religieuse qui constitue le centre même et pour ainsi dire le levier de commande de toute la contestation anti-hégélienne ${ }^{112}$; tandis que la dialectique abstraite du Magister Adler représente tout le contraire d'une intériorité rappelant une subjectivité passionnée en étroit rapport avec l'infini.

En outre, et parce qu'il a été formé par la philosophie hégélienne, Adler met l'accent sur les notions de système spéculatif et d'objectivité. Toute son affaire revient par conséquent, pour reprendre une expression qu'il emploie dans l'avant-propos

109. Ibid., p. 214.

110. Carignan, Maurice. Individu et société chez Kierkegaard. Collection dirigée par D. Braybrooke, publication pour The Canadian Association for Publishing in Philosophy par Dalhousie University Press, Halifax, Nouvelle-Écosse, prem. éd., 1977, p. 11.

111. Livre sur Adler, O.C., XII, p. 153.

112. Carignan, Maurice. Individu et société chez Kierkegaard, p. 9. 
de ses Sermons, à " expliquer le christianisme ", fidèle en cela à la tendance spéculative de son époque et à l'apologétique qui veut rendre tout vraisemblable, pourrie qu'elle est de philosophie rationalisante ${ }^{113}$. Dans Le livre sur Adler, Kierkegaard remarque à ce sujet que « l'on s'est efforcé, sous des prétextes divers, dont le dernier est la spéculation, de rendre le christianisme vraisemblable, intelligible et de l'éliminer de la langue divine du paradoxe pour le transposer en platt-deush de spéculation ou de vulgarisation ${ }^{114}$. Ces procédés, à son avis, sont totalement contraires à l'esprit du christianisme dont l'essence consiste plutôt dans un paradoxe qui défie la pensée. "La foi, écrit-il, correspond très exactement à la notion du prodigieux, de l'absurde, de l'invraisemblable, de ce qui est folie pour la raison ${ }^{115}$, aussi est-il parfaitement indifférent de savoir si ce qui va, pour ainsi dire, « contre la pensée », est pensable. Aussi toute apologie du christianisme consciente de son but devraitelle recourir à la dialectique qualitative pour soutenir à fond l'invraisemblance du christianisme et permettre l'apparition d'un renouveau ${ }^{116}$. Autant l'éloquence grecque a-t-elle reposé sur la vraisemblance (Aristote), autant l'éloquence chrétienne, écrit Kierkegaard dans son Journal, doit-elle reposer sur l'invraisemblance ${ }^{117}$. Adler n'y a rien compris, ayant tenté de tout rendre vraisemblable (il a fallu qu'il aille jusqu'à « faire l'exégèse de sa propre révélation selon la méthode qui a servi à l'exégèse et à la dogmatique rationnalistes pour interpréter la révélation du christianisme ${ }^{118}$ ), et ne s'étant pas tenu à l'impossible, c'est-à-dire au fait-paradoxe qu'eût été l'apostolat. C'est donc parce que le paradoxe, en son essence, est précisément la protestation élevée contre l'immanence et que les explications d'Adler n'ont jamais plafonné au-delà de la sphère immanente,

113. Journal (extraits, 1846-1849), tome II, note 3 des traducteurs, p. 144. Ailleurs on peut lire ces quelques lignes critiques de la plume de Kierkegaard : "C'est comme une épigraphe de toute la façon moderne de prêcher le christianisme, ce mot de Thalès : on doit dire le vraisemblable, mais taire l'impossible. Plutarque. Le Banquet des sept sages, chap. 17. (et en marge :) Car on a mis en effet tout le christianisme dans le vraisemblable . . . pour le taire ". Journal (1848), tome II, p. 306. IX A 239.

114. Livre sur Adler, O.C., XII, p. 77.

115. Ibid., p. 87.

116. Ibid., p. 77.

117. Journal (1845), VI A 19.

118. Livre sur Adler, O.C., XII, p. 114. 
qư'on peut dire qu'il n'a pas saisi ce qu'il y avait d'invraisemblable dans le christianisme.

Le cas du Magister Adler, faut-il dire, est unique : « il est l'hömme qui a reçu la révélation et en mêmê temps l'exégète " et « c'est justement pourquoi il dévoile à merveille le fond de la tendance moderne $"{ }^{i 19}$. Et Viallaneix écrit :

Il se laišse entraîner par la spéculation, qui ešt de mode, pour déguiser en ùn doute concernant les vérités religieuses sa trop réelle désobéissance viśs-à-vis de l'autorité, pour confondre à son ävantage, la sphère intellectuelle ou métaphysique et la sphère éthico-religieuse, où s'exerce la volonté et se prend là " grande décision ". La spéculation, en effet, fait mine de prendre au sérieux les problèmes d'exéégèse, d'adopter une attitude scientifique devant l'Écriture Sainte. Màis ce sont là des astuces, qui visent seülement à dispenser d'obéir: Pascal dénonçait cette dérobàde, en affirmant que la "difficulté de croire n'était souvent que la difficulté d'obéir ${ }^{120}$.

On en revient à cet " art du raisonnement qui permet à l'homme de se tromper " ${ }^{121}$, comme l'écrit Saint-Exupéry dans Citadelle, et qui conduit au secret de la confusion religieuse d'Adler : son insoumission. Sous cet aspect, Adler suit fidèlement la courbe régulière de la spéculation moderne : " On a honte d'obéir au roi parce qu'il est le roi - alors on lui obéit PARCE QUEE il est intelligent. On a honte d'obéir à Dieu parce qu'il est Dieu, alors on lui obéit . . . PARCE QUE il est un très grand génie, peut-être presque le plus grand, encore plus grand que Hegel . . . . ${ }^{122}$. La satire vise donc à démontrer que l'obéissance ne doit pas être conditionnée par une autorité transitoire, fondée sưr des règles intellectuelles, éthiques ou même logiqués, mais qu'elle doit ressortir de la prise de conscience directe d'une

119. Ibid., p. 120. Remarque *. Souvenons-nous qu'Adler, parlant de sa révélation, a émis le souhait " d'en développer le contenu chrétién sous une forme plus adéquate » avanit de considérer, dans sa dernière lettre, sès "Sermons (ou ses Études) ainsi que dés catégories dogmatiques dont il use fréquemment comme des points de repère indispensables à son exaltation naissante . . . " Or Kierkegàard ajoute ici une seconde remarqúe ** à la première : "Quiconque possède quelques notions relatives à une certaine théologie et exégèse modernes et sait que l'on a traité l'Écriture Sainte d'œuvre spontanée, inspiration première que l'oń espère perfectible, etc. tout en lui conservant le nom de révélation, retrouvera ces idées tout au long de l'argumentation du Mag. Adler ".

120. Viallaneix, Nelly, Kierkegaard, propbète de la modernité, p. 374.

121. Saint-Exüpéry, Citádelle, nouvelle édition Gallimard, Collection Folio, c1948, p. 92.

122. Journal '(extraits 1846-1849), tome II, p. 177. VIII A 436. 
« qualité spécifique venue d'ailleurs » et s'imposant inconditionnellement à l'individu sous la forme d'une autorité divine. «Ce n'est pas, comme l'enseigne la philosophie confuse de nos jours, le contenu de la doctrine qui importe . . . mais le fait de la révélation et ce qui en découle : l'autorité divine ${ }^{123}$. Dès l'instant où, comme Adler, influencé par une philosophie spéculative, l'on cherche à incorporer totalement le fait de la révélation à la réflexion, on aboutit d'une façon ou d'une autre à un " domaine impénétrable " ou à une " contradiction interne " qui finit par amoindrir le "facteur qualitatif décisif », le fait déterminant qu'est l'autorité de Dieu ${ }^{124}$. Et, inévitablement, la réflexion conduit alors à adopter la position sécuritaire du génie, position de désobéissance à Dieu, contraire à celle de l'apôtre.

Mais il est possible d'envisager l'affaire sous un angle différent. Posons, pour commencer, que l'erreur fondamentale de la philosophie hégélienne est d'avoir supprimé la catégorie de l'Individu pour poser celle de la génération ${ }^{125}$. Jean Brun souligne notamment que Kierkegaard considère que "le hégélianisme est une sorte de paganisme réduisant l'homme à une espèce animale douée de raison dans laquelle, précisément, l'Individu est toujours inférieur à l'espèce " ${ }^{126}$. Adler, en tant qu'hégélien, ne pouvait donc échapper à l'idée de la supériorité du peuple ou de la génération sur son individualité. Il peut bien dire, s'il veut, qu'il n'est plus hégélien, il demeure néanmoins en plein dans la dialectique de justification de l'hégélianisme : le philosophe dit que le peuple est la vérité ; ergo, Adler croit que l'approbation du peuple est nécessaire. Parce que le peuple a entendu sa cause, Adler peut « désormais prétendre qu'il a eu vraiment une Révélation puisque la foule lui aura confirmé le bien-fondé d'une conviction qui, jusque-là, n'était que subjective " ${ }^{127}$. À cela, et pour être conforme, malgré lui, à la doctrine hégélienne de l'identité d'intérieur et d'extérieur, on doit ajouter qu'Adler a brûlé tous ses manuscrits inspirés du hégélianisme afin de manifester avec éclat qu'il a rompu de façon décisive

123. Livre sur Adler, O.C., XII, Annexes IV, Autre rédaction du Chapitre I, p. 248.

124. Ibid., p. 248.

125. Compte rendu littéraire, O.C., VIII, p. 204.

126. Ibid., Introduction de Jean Brun, p. xxiv.

127. Livre sur Adler, O.C., XII, Introduction de Jean Brun, p. xx. 
avec la philosophie hégélienne. Il voulait montrer que sa résolution intérieure correspondait effectivement à sa décision extérieure ; en fait, il agissait en vertu d'une doctrine hégélienne, celle qui tend, précisément, à affirmer que l'homme est ce qu'il fait $^{128}$. Cependant, si Adler avait été le moindrement sérieux, au sens d'un rapport avec Dieu, il aurait caché plutôt sa décision, n'aurait pas attendu qu'on partage autour de lui son avis pour se sentir convaincu ; il se serait mis à l'épreuve dans le silence de son intériorité afin de voir s'il n'était pas dupe d'une illusion et si, dans sa faiblesse, il ne cherchait pas sa force dans une décision d'ordre extérieur ${ }^{129}$.

Telle est, en définitive, la satire que Kierkegaard fait indirectement de la philosophie hégélienne à travers Adler, en montrant que sa confusion n'a pas seulement eu lieu à la suite d'un choc émotif et d'un manque de formation dogmatique religieuse, mais qu'elle a été produite aussi par sa formation philosophique inconciliable sur tous les plans avec la théologie chrétienne. Telle est, également, l'explication philosophique qu'il nous donne de la façon dont un pseudo-génie peut finir par se prendre pour un véritable apôtre.

Notons cependant que le chapitre IV du Livre sur Adler n'a pas pour seul but de considérer uniquement le cas Adler comme satire de la "philosophie hégélienne » mais aussi comme satire du «temps présent ${ }^{130}$ ou de la « chrétienté actuelle ». Car nul mieux que le Magister Adler n'illustre en effet aussi bien

. . . la confusion totale où nous sommes parvenus depuis que la chrétienté est devenue un titre et que dans un pays CHRÉTIEN où TOUS SONT CHRÉTIENS l'on peut être licencié en théologie et même devenir pasteur sans avoir seulement effleuré le problème qui consiste à se demander, en conscience, si l'on est soi-même chrétien. ${ }^{131}$

En ce sens, croit Kierkegaard, " aucun de mes contemporains ne répond aussi strictement à l'appellation de "phénomène » qu'Adler ${ }^{132}$, parce que sa confusion religieuse est partagée par

128. Carignan, Maurice. Individu et société chez Kierkegaard, p. 14. Explications données sur la théorie hégélienne d'identité et la critique qu'en fait Kierkegaard.

129. Livre sur Adler, O.C., XII, p. 185-186.

130. Ibid., p. 175.

131. Ibid., p. 39.

132. Ibid., p. 89. 
toute son époque. D'où le titre de ce deuxième projet d'avantpropos daté de 1847 : "La confusion religieuse de notre époque éclairée par le Magister Adler en tant que phénomène " ${ }^{133}$. Comme beaucoup, il est vrai, Adler « donne tout à fait l'impression d'un païen soudain mis en contact avec le christianisme ${ }^{134}$. Car il se croyait bel et bien chrétien, comme tous les autres, mais cela ne l'a pas empêché de verser dans l'illusion. Aussi pour Kierkegaard l'événement survenu dans la vie du Magister est-il un « mémento » à l'adresse de toute la chrétienté géographique ${ }^{135}$.

En somme, c'est surtout la confusion religieuse de l'époque moderne qui est éclairée ici par le phénomène Adler. Mais ce n'est guère la première fois que Kierkegaard traite de cette confusion des temps modernes ; il en avait déjà recherché les causes dans son Compte rendu littéraire ${ }^{136}$, daté de 1846 , et il continuait de les poursuivre lorsqu'il écrivit, en 1847, la Dialectique de la communication éthique et éthico-religieuse ${ }^{137}$, ouvrage qui ne fut publié qu'après sa mort. Or démêler les liens qui existent entre ces deux derniers livres et Le livre sur Adler, lequel fut commencé, on se le rappellera, en 1846, ne constituerait pas qu'une entreprise originale, mais présenterait aussi, dans la perspective d'une continuité dans l'application de la dialectique kierkegaardienne au cas du Magister Adler, une étude fort avantageuse. C'est ainsi que, s'il fallait exposer sommairement les principaux liens qui peuvent exister entre le Compte rendu littéraire et Le livre sur Adler, nous dirions qu'Adler, d'une part en raison de son bavardage et de sa spéculation, et d'autre part, en raison de son manque d'intériorité dû à la dialectique trop abstraite de son existence, est le parfait représentant de son époque, laquelle a aussi supprimé la disjonction essentielle que Kierkegaard conçoit entre objectivité et subjectivité. Ainsi Adler partagerait-il à maints égards l'insoumission de ses contemporains vis-à-vis de l'autorité divine. En outre, il faut ajouter que l'époque moderne a également mis de l'avant la catégorie de génération, catégorie à

133. Ibid., Annexes 2. Premier projet d'avant-propos, p. 233.

134. Ibid., p. 226.

135. Ibid., p. 217.

136. Compte rendu littéraire, O.C., VIII.

137. Dialectique de la communication éthique et étbico-religieuse, O.C., XIV. 
laquelle Adler est loin d'être indifférent, du fait qu'il va chercher auprès du public la confirmation de sa propre révélation et qu'il ressent, si vivement, le besoin de se communiquer à lui, le plus vite possible et sur le plan esthétique (il fait de sa révélation un prétexte à production littéraire ; on le voit aux quatre derniers ouvrages qu'il publie d'un coup en 1846), sans la moindre considération pour le contenu éthico-religieux de sa présumée expérience. Et sous ce rapport on peut dire qu'il a sacrifié à la génération le meilleur de son individualité. Bref, Adler et son époque ont, pour finir, littéralement aboli la différence qualitative essentielle entre Dieu et l'homme, entre l'apôtre et le génie, entre l'autorité venue d'ailleurs et celle venant d'ici. Adler est par conséquent le parfait représentant des temps modernes, précisément en ce sens que, ayant reçu sans grande passion une éducation d'essence trop raisonnable, mais déficiente pour ce qui est de la connaissance qu'elle apportait du contenu dogmatique du christianisme, il a sombré comme ses contemporains dans la confusion religieuse la plus totale, au point d'en oublier de devenir chrétien.

Quant aux liens qu'on peut faire entre la Dialectique de la communication éthique et éthico-religieuse et Le livre sur Adler, on peut dire, pour commencer, qu'il est évident que le type de communication employé par Adler dans ses nombreux ouvrages n'est pas idéal du strict point de vue éthico-religieux, ceci à cause même de son fondement, qui fait davantage penser à de confuses exaltations subjectives qu'aux faits réellement objectifs de la venue du Sauveur et de la Révélation sur lesquels doit reposer toute communication dialectique véritablement chrétienne. C'est donc en raison même de son manque de savoir conceptuel religieux, condition préalable à toute communication éthico-religieuse, que le Magister Adler n'entre dans aucune des normes de communication idéales à ce point de vue, définies dans la Dialectique de la communication éthique et éthico-religieuse. Le type de communication qu'il emploie le plus volontiers n'est pas celui de la communication indirecte, mais celui de la communication directe, alors qu'il s'efforce de se maintenir au niveau d'une stricte communication objective de savoir. On le remarque aisément à ce qu'il réduit sa présumée doctrine à des " catégories dogmatiques » qui lui servent de simples « points de repère ». 
C'est de sa propre pensée dont il est question, c'est-à-dire uniquement d'une communication de lui-même, à sens unique, et à travers un enseignement magistral qui ne laisse aucune place à la communication indirecte, sur le plan de l'existence, de la vérité paradoxale et de sa puissance éthico-religieuse.

Cependant Adler est l'enfant de son siècle : il manque pardessus tout d'éducation. Les temps modernes ont, en effet, plus forcé sur " l'instruction » que sur « l'art d'éduquer » (qui est, dans l'acceptation antique de ce terme, un développement harmonieux du support des divers dons, talents et dispositions naturelles de la personnalité éthique au sens du caractère) ou que sur l'instruction réellement chrétienne. On pensait, sans doute, que, si toutes les mesures sont prises pour assurer l'enseignement de l'enfant, il s'éduquera bien tout seul pour le reste. Or c'est évidemment ce type de mentalité qui conduit à des catastrophes du genre de celle qui est arrivée dans la vie du Magister Adler. Car si Adler, par exemple, avait reçu une stricte éducation éthicoreligieuse et une formation chrétienne adéquate, c'est-à-dire une impression décisive du spécifique chrétien, il aurait su contenir son exaltation subjective en enfermant l'impetus de son émotion dans un langage conceptuel qui lui aurait servi d'appui. En outre, il y aurait eu chez lui une assimilation existentielle réelle (réduplication) qui l'aurait retenu d'agir ainsi à la légère, en se fiant au seul résultat de son examen de théologie . . . Car c'est sous cet aspect qu'Adler, précisément, est le représentant de son époque, tant par sa façon directe de communiquer, lui qui, par son bavardage, a supprimé la disjonction essentielle entre la communication directe-indirecte de la vérité éthico-religieuse et la communication directe, que par son manque sérieux d'éducation éthico-religieuse.

\section{***}

Aux yeux de Kierkegaard l'événement survenu dans la vie du Magister Adler fait, pour ses contemporains, office de rappel $^{138}$. Il montre à quel point la confusion religieuse de celuici est représentative de celle de son époque, en ce sens qu'elle

138. Livre sur Adler, O.C., XII, p. 227. 
révèle indirectement « comment on peut, dans la chrétienté géographique, être chrétien et même devenir prêtre chrétien sans avoir la moindre impression du christianisme, au sens de : devenir chrétien ${ }^{139}$.

Mais Le livre sur Adler dans sa totalité n'explique-t-il pas aussi l'illusion religieuse fondamentale de son époque par une confusion générale des sphères, d'où serait venu le projet d'éditer, en même temps que le Compte rendu littéraire des Deux âges, un « Compte rendu littéraire des écrits d'A.P. Adler " $?^{140}$ Une confusion générale des sphères, tel serait certes le point de vue qui donnerait le plus de recul pour analyser la double confusion d'Adler et de son temps. À cet égard, la confusion la plus universelle, si l'on peut dire, dégagée par Le livre sur Adler, est celle entre la sphère transcendante et la sphère immanente, lesquelles embrassent toutes les autres confusions. Entre les deux, en effet, une différence qualitative essentielle au point de vue du christianisme vient jeter sa différence radicale : - celle qui existe entre la Révélation chrétienne et la pensée humaine, entre l'apôtre et le génie, entre l'autorité divine et l'autorité transitoire (l'une reposant sur la grâce surnaturelle et sur le mystère du paradoxe, l'autre sur la nature et sur l'intelligence), celle qui existe, enfin, entre Dieu, sagesse incommensurable juchée dans l'éternité, et les hommes, plus ou moins égaux dans leurs rapports entre eux en tant qu'hommes. Car, dans l'esprit de Kierkegaard, hors de la connaissance de cette différence qualitative éternelle donnée par la foi et par une stricte formation conceptuelle chrétienne, il n'est point de salut, mais seulement une immense confusion générale qui absorbe, nivelle, égalise et empêche le saut passionné de l'individu vers l'infini. C'est alors que s'entremêlent l'universellement humain (la morale) et l'éthique religieuse, confondant les sphères émotives et intellectuelles (esthétique, poétique ; métaphysique, philosophique, etc.) avec la sphère transcendante du paradoxe. Insidieusement, la religion sort diminuée de ce mélange : la révélation de l'apôtre a été réduite au strict contenu intelligible et à une exaltation subjective ; la

139. Ibid., p. 227.

140. En 1846, Kierkegaard se proposait d'intituler sa première version du livre : Compte rendu littéraire. Le Mag. Adler, étude psychologique d'après nature, c'est-à-dire d'après ses écrits, par Sopren Kierkegaard. Ibid., Avertissement de l'éditeur, p. xxiv. 
médiation, comme dans la dialectique hégélienne, a relativisé le paradoxe ; le doute et l'insoumission de la pensée moderne ont finalement pris le pas sur l'obéissance inconditionnelle à l'autorité divine. Il n'y a plus moyen de communiquer l'existence, de donner à l'individu une éducation telle qu'il puisse accueillir généreusement le souffle de l'Esprit et se comprendre en lui au moyen de déterminations religieuses correspondantes, parce que la communication directe-indirecte, surtout indirecte, de la vérité éthico-religieuse a été abolie. Ainsi, de toutes les manières possibles, la confusion générale des sphères a-t-elle profondément altéré la différence qualitative essentielle entre Dieu et les hommes, entre le christianisme et Adler.

Mais encore, ce ne sont guère ceux qui, comme Feuerbach, cherchent à abolir totalement la religion qui sont vraiment à l'origine de cette confusion, car ceux-là au moins sont reconnaissables à leur position clairement définie, en dehors de toute religiosité $^{141}$. Non, pour le penseur danois, ceux qui sont le plus à craindre, ce sont ceux qui, à la manière d'Adler, n'en faisant qu'à leur tête et voulant gouverner, viennent s'immiscer et semer le doute et l'insubordination à l'intérieur du monde religieux. Ces pasteurs confus de la confusion sont les plus à redouter justement parce qu'ils sont moins motivés à rechercher, en toute honnêteté, le donné authentique du religieux, qu'à faire passer d'abord leur volonté de puissance, et avec elle, malheureusement, leur savoir abstrait et désordonné, puisé partout dans l'amalgame des sphères. Aussi Kierkegaard juge-t-il que l'attitude de l'Église danoise, dans cette affaire, a été irréprochable, que c'est même avec beaucoup de perspicacité et de cohérence que les membres de la commission d'enquête ont mené l'interrogatoire d'Adler ${ }^{142}$.

Mais en ce cas pourquoi Kierkegaard n'a-t-il pas publié Le livre sur Adler? Il y a plusieurs explications. L'éditeur, notamment, nous signale que Kierkegaard " ne voulait pas accabler davantage Adler dans son conflit déjà compliqué avec l'autorité établie ", qu'il craignait, d'autre part, " que la grosseur de l'ouvrage et sa référence constante à Adler n'en rende la lecture trop

141. Ibid., Annexes 2. Deuxième projet d'avant-propos, p. 235.

142. Ibid., p. 127. 
fastidieuse » et qu'il désirait, enfin, « tenir compte (. . .) des réactions présumées de ses propres lecteurs $"{ }^{143}$. Quant à nous, nous avancerions une quatrième explication. Sand doute Kierkegaard veut-il tenir compte à la fois d'Adler et dé ses lecteurs, sans doute approuve-t-il la réaction des autorités ecclésiastiques, mais il sait également, ainsi que nous le fait remarquer André Clair, que « l'attitude de l'Église, tout irréfutable qu'elle soit manque pourtant le fond de la question, à savoir le vrai rapport à l'Exception " ${ }^{144}$. Car Adler n'a pas été destitué parce qu'il s'est prévalu, au plan spirituel, d'une révélation, mais bien à cause du trouble de son esprit, plus exactement, à cause de son incapacité à prouver, sur le plan immanent, la cohérence de sa position. Dans le cas d'un véritable apôtre, l'Église d'État n'aurait d'ailleurs pas eu besoin d'agir de cette manière, car le véritable apôtre aurait été de lui-même amené à la quitter. "C'est, nous l'avons dit, écrit Kierkegaard, un non-sens que de vouloir enseigner comme apôtre dans l'Église établie. Quiconque s'entend à la dialectique qualitative le comprendra sans peine ${ }^{145}$. Mais, en raison de cette impossibilité de coexistence entre l'apôtre et l'Église, ne serait-il pas permis d'avancer qu'en ne publiant pas Le livre sur Adler, dont le contenu remet autant en question l'Église d'État que les illuminés, Kierkegaard se soit en quelque sorte ménagé une porte de sortie ? Ce ne sera en effet qu'à partir de 1845 que le penseur danois s'en prendra plus directement à l'Église existante, à l'occasion d'une polémique, devenue célèbre aujourd'hui, qui l'opposera au successeur de l'évêque Mynster (voir notre note, no 4). Kierkegaard, qui savait que l'Église établie altérait le contenu éminemment paradoxal et spécifiquement religieux du christianisme, aurait donc senti que celleci n'était pas prête à rencontrer un véritable apôtre ou quiconque prétendant connaître la spécificité d'une telle autorité transcendante.

Remarquons cependant qu'il s'agit aussi d'une attitude très contemporaine, comme le signale un peu cette boutade, certes

143. Ibid., Avertissement de l'éditeur, p. xxv. Voir aussi Papirer VIII 1 A 164, VIII 2 et VII 2.

144. Clair, André. "Le Général, l'Individu, l'Exception. Kierkegaard et le pasteur Adler ", p. 219.

145. Livre sur Adler, O.C., XII, p. 126. 
irrespectueuse et bien paradoxale, que Bernanos a mise sur les lèvres du doyen dans son Journal d'un curé de campagne : "Dieu nous préserve des saints ${ }^{146}$ ! C'est pourquoi il convient de souligner ici, par fidélité à la pensée de Kierkegaard, l'actualité du Livre sur Adler, lequel, au-delà de toutes les spéculations et de toutes les autorités transitoires et immanentes, nous invite surtout au rapport avec une vérité vivante, mystérieuse, éternelle et transcendante. Car c'est de la même façon qu'éclate dans $L e$ livre sur Adler toute l'illusion de la chrétienté, et celle, évidemment, de son époque, à laquelle il ne restait, selon le penseur danois, qu'une seule et unique solution : l'éducation religieuse ${ }^{147}$. C'est bien à cette tâche que Kierkegaard s'est attelé, lui dont " l'objet de toute la production est, dans la chrétienté, devenir chrétien " ${ }^{148}$. Deux ans après le commencement de sa rédaction du Livre sur Adler, Kierkegaard proposait à nouveau dans le Point de vue explicatif de mon cuvre d'écrivain : "La tâche à proposer à la plupart dans la chrétienté est la suivante : partant du <poète> ou de la vie conforme à ses conceptions, partant de la spéculation, ou d'une vie consacrée dans l'imaginaire (. . . à la spéculation (au lieu d'exister), partant de là, devenir chrétien ${ }^{149}$. "Devenir chrétien ", quelle abstraction ! - mais c'est bien là l'essence de toute l'œuvre. On voit alors en quoi Le livre sur Adler n'a été qu'une esquisse faite "d'après nature » de cette invitation à se mettre à L'École du Christianisme que Kierkegaard acheva en 1850, partant, à se mettre à l'écoute d'un dialecticien du christianisme, lequel, en écartant sans cesse toutes les illusions, est le seul à pouvoir exposer la doctrine en la vissant pour ainsi dire dans le présent ${ }^{150}$.

Certes, Kierkegaard n'est évidemment pas le seul à prêcher un rapport direct de l'Individu avec l'Absolu, à faire voir un ordre transcendant ; des comparaisons intéressantes pourraient être faites avec des auteurs aussi contemporains que, par exem-

146. Bernanos, Georges. Journal d'un curé de campagne. Paris, Librairie Plon, c1936, p. 7677.

147. Dans le Point de vue explicatif sur mon auvre d'écrivain, par ailleurs écrit en 1848, c'està-dire deux ans après que Kierkegaard ait entrepris la rédaction du Livre sur Adler, on peut lire cette remarque : "Si je devais d'un mot me prononcer sur mon époque, je dirais qu'elle manque d'éducation religieuse ". O.C., XVI, p. 52.

148. Ibid., p. 64.

149. Ibid., p. 52.

150. Journal (extraits 1846-1849), tome II, p. 267. IX A 114. 
ple, Saint-Exupéry ou Gabriel Marcel, ou comme avec Max Scheler (Le Saint, le Génie, le Héros). Mais, par delà toutes les formes d'étude, par delà même les contradictions des doctrines et des appartenances religieuses, l'essentiel demeure encore cette quête primitive, inconditionnelle du Royaume de Dieu. Car en effet quel siècle, aussi civilisé soit-il, peut-il se passer d'une telle science intérieure, de tels maîtres spirituels, de tels apôtres? L'idéalisme véritable, le seul, commence lorsqu'on a oublié ces vérités premières.

Département de philosophie Université d'Ottawa 